\title{
Models that link and suggest data about elementary particles, dark matter, and the cosmos
}

Thomas J. Buckholtz

Ronin Institute for Independent Scholarship, Montclair, New Jersey 07043, USA

\begin{abstract}
We suggest progress regarding the following six physics opportunities. List all elementary particles. Describe dark matter. Explain ratios of dark matter to ordinary matter. Explain eras in the history of the universe. Link properties of objects. Interrelate physics models. We use models based on Diophantine equations.

Keywords: beyond the Standard Model, dark matter, evolution of the universe, cosmological tensions, galaxy formation, cosmic inflation, neutrino masses
\end{abstract}

\section{Contents}

1 Introduction 2

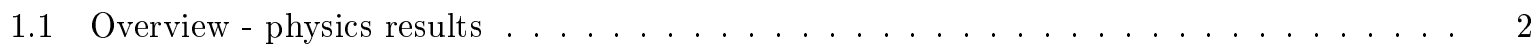

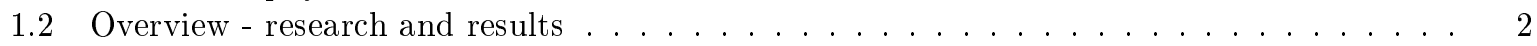

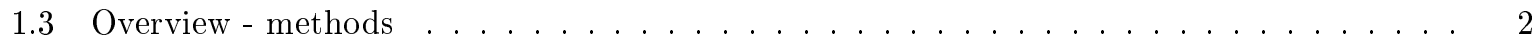

1.4 Relationships between our work and other work $\ldots \ldots \ldots \ldots \ldots \ldots$

\begin{tabular}{|lll}
2 & Methods & 9
\end{tabular}

$2.1 \quad$ Charge, mass, other properties, and long-range forces . . . . . . . . . . . . . . . 10

2.2 Elementary particles that do not carry long-range forces $\ldots \ldots \ldots \ldots \ldots$

2.3 Isomers and dark matter $\ldots \ldots \ldots \ldots \ldots \ldots$

\begin{tabular}{ll|l}
\hline & Results & 18
\end{tabular}

$3.1 \quad$ Explanations and suggestions regarding elementary particles . . . . . . . . . . . . . . . 18

3.2 Explanations and suggestions regarding dark matter $\ldots \ldots \ldots \ldots$. . . . . . . . 19

3.3 Explanations and suggestions regarding the rate of expansion of the universe $\ldots \ldots \ldots 23$

3.4 Explanation regarding data about large-scale clumping . . . . . . . . . . . . . . . 24

3.5 Explanation regarding data about interactions between neighboring galaxies . . . . . . . . 24

3.6 Suggestions regarding galaxy formation $\ldots \ldots \ldots \ldots \ldots \ldots \ldots$

3.7 Explanations regarding dark matter data $\ldots \ldots \ldots \ldots \ldots \ldots$. . . . . . . . . 26

3.8 Explanation regarding data - other than dark matter data - about galaxies $\ldots \ldots \ldots . \quad 26$

\begin{tabular}{ll|l}
4 & Discussion & 26
\end{tabular}

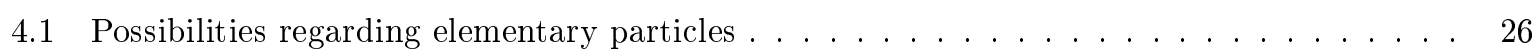

4.2 Possibilities regarding constraints regarding dark matter $\ldots \ldots \ldots \ldots \ldots \ldots$

4.3 Possibilities regarding interactions involving the jay boson $\ldots \ldots \ldots \ldots \ldots \ldots$

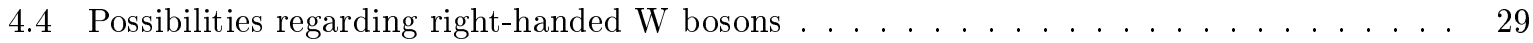

4.5 Relationships between our modeling and other modeling . . . . . . . . . . . . . . . . 29

$\begin{array}{lll}5 \text { Conclusions } & 30\end{array}$

\begin{tabular}{|ll}
\hline Acknowledgments & 30
\end{tabular}

\begin{tabular}{ll}
\hline Funding statement & 31
\end{tabular}

Email address: Thomas.Buckholtz@RoninInstitute.org (Thomas J. Buckholtz) 


\section{Introduction}

This unit previews physics results we propose, previews methods we use, and relates our work to other work in elementary particles, astrophysics, and cosmology.

\subsection{Overview - physics results}

This essay pursues the following two challenges. Describe new elementary particles and dark matter. Use descriptions of elementary particles and dark matter to explain astrophysics data and cosmology data.

Our explanations regarding large-scale data might help validate our descriptions of possible new elementary particles and our description of dark matter.

\subsection{Overview - research and results}

Figure 11 diagrams flow - from other people's work through results that this essay suggests - regarding our research. Our work has roots in the known elementary particles and in concordance cosmology. One basis for our work features a hypothesis that nature includes six isomers of known elementary particles - of which only one isomer associates with ordinary matter and five isomers associate with most dark matter. Another basis for our work features new modeling based on Diophantine equations. We suggest new elementary particles and a specification for dark matter. We suggest insight regarding modeling gravity and regarding galaxy formation. We suggest explanations for known data for which - seemingly - other modeling does not offer explanations. We suggest data about aspects of the universe - that people might be able to verify or refute.

Figure 2 alludes to all known elementary particles and to elementary particles that our work suggests. (Perhaps, preview table 9.)

Figure 3 suggests an explanation for the known ratio - five-plus to one - of dark matter density of the universe to ordinary matter density of the universe. (Perhaps, preview table 7 and table 18 ,) The figure has bases in our specification for dark matter. The specification has roots in our hypothesis that associates with six isomers of known elementary particles. Each isomer of known elementary particles associates - approximately - with its own photon. Each isomer of elementary particles associates with its own $0.5 \mathrm{~N}^{\prime}, 0.5 \mathrm{R}, 0 \mathrm{I}$, and $1 \mathrm{~J}$ elementary particles.

Figure 4 suggests eras in evolution of the universe. (Perhaps, preview table 15.) As far as we know, direct observations and data associate only with the two multi-billion-years eras.
Figure 5 suggests eras in the evolution of - and various ratios of dark matter to ordinary matter for - galaxies. (Perhaps, preview table 17 and table 18.) People have observed galaxies that associate with each one of the suggested approximate ratios - one to zero-plus, five-plus to one, four to one, and zero-plus to one.

Figure 6] suggests possible relationships between physics properties. (Perhaps, preview table 10 and table 12.) This essay leaves open possible opportunities to use these possible relationships to envision possibly more - than this essay explores - fundamental aspects of nature.

Figure 7 suggests possible rest energies for all known elementary fermions (including neutrinos) and all suggested elementary fermions. (Perhaps, preview table 11 table 12 , and table 13 .) This essay associates each rest energy with direct use of or extrapolation from a few formulas. (Perhaps, preview table 12 and table 13.$)$

\subsection{Overview - methods}

One goal of our modeling is to match and extend a list of properties - of objects - that people infer or might infer based on observations based on so-called long-range interactions (or, so-called longrange forces). Long-range interactions include electromagnetism (which associates with notions of a spin-one boson - the photon), gravity (which associates with notions of a yet-to-be-found spin-two boson - the graviton), possibly interactions that would associate with a spin-three boson, and possibly interactions that would associate with a spin-four boson.

We find it convenient to divide elementary particles into three sets - carriers of long-range interactions, other elementary bosons, and elementary fermions. We associate, respectively with the three sets, the symbols LRI (as in long-range interaction or as in elementary boson that associates with a long-range interaction), SRI (as in short-range interaction or as in elementary boson that does not associate with a long-range interaction), and ELF (as in elementary fermion).

We develop mathematics modeling that outputs characteristics of long-range interactions and properties - of objects - that long-range interactions measure. The modeling features solving Diophantine equations.

LRI solutions come in pairs. For example, regarding electromagnetism, one so-called PROP solution associates with the property of charge. That PROP solution has a so-called CURR partner solution that associates with a current of charge. We think that each LRI pair of one PROP solution and 


\section{Research - elementary particles, dark matter, and the cosmos}

\begin{tabular}{|c|}
\hline Starting points \\
Known elementary particles \\
Concordance cosmology \\
\hline Isomers hypothesis \\
(six isomers of non-long-range- \\
force elementary particles) \\
\hline Diophantine modeling \\
(regarding long-range forces and \\
other elementary particles)
\end{tabular}
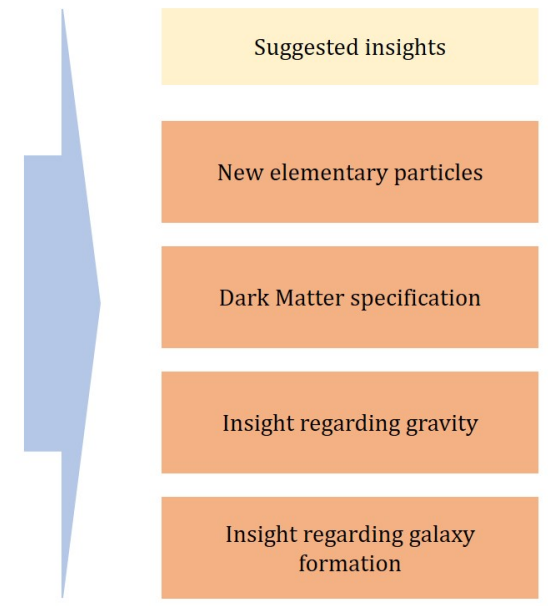

Copyright (C) 2021 Thomas J. Buckholtz

Associations regarding nowknown and possible-future data

Explain ratios of Dark Matter effects to Ordinary Matter effects

Explain eras in the history of the universe

Resolve "tensions" between cosmology data and concordance cosmology

Suggest eras that would precede cosmic inflation

Figure 1: Research flow - from roots to results.

\section{Elementary particles}

(known and possible)

Copyright (C) 2021 Thomas J. Buckholtz

\begin{tabular}{|c|c|c|c|c|c|c|c|c|c|}
\hline \multirow[t]{4}{*}{ S, m : } & $0,>0$ & $0,=0$ & $0.5,>0$ & $1,>0$ & $1,=0$ & $2,=0$ & $3,=0$ & $4,=0$ & \multirow[b]{2}{*}{$\mathrm{Fr}$} \\
\hline & \multirow[t]{3}{*}{$0 \mathrm{H}$} & & $0.5 \mathrm{C}_{1}(3), 0.5 \mathrm{~N}(3), 0.5 \mathrm{~N}^{\prime}(3)$ & $1 \mathrm{~W}_{1}, 1 \mathrm{Z}$ & & & & & \\
\hline & & & & & $1 \mathrm{G}$ & $2 \mathrm{G}$ & $3 G$ & $4 \mathrm{G}$ & Fr/Irlc \\
\hline & & 0I & $0.5 Q_{2 / 3}(3), 0.5 Q_{1 / 3}(3), 0.5 R(3)$ & & 1J, $1 \mathrm{U}(8)$ & & & & Irlc \\
\hline
\end{tabular}

Legend:

$S-\operatorname{spin}$ in units of $\hbar$. $\left\{\mathrm{S}\right.$ as in $\left.\mathrm{S}(\mathrm{S}+1) \hbar^{2}\right\}$

$\mathrm{S}, \mathrm{m}-$ spin in units of $\hbar$, mass [ $>0$ denotes positive. $=0$ denotes zero.].

$S \Phi_{\mid(\text {charge)/(charge of the electron)] [if nonzero] }}$ (Number of particles [if more that one particle]).

$0 \mathrm{H}$ - Higgs boson. $0.5 \mathrm{C}$ - charged leptons. $0.5 \mathrm{~N}$ - neutrinos. $0.5 \mathrm{~N}^{\prime}$ - heavy neutrinos. $1 \mathrm{~W}-\mathrm{W}$ boson. $1 \mathrm{Z}-\mathrm{Z}$ boson.

$1 \mathrm{G}$ - photon. $2 \mathrm{G}$ - graviton. $3 \mathrm{G}$ - to be named [interacts with a function of elementary-fermion flavour]. $4 \mathrm{G}$ - to be named [interacts with angular momentum $\mathrm{S} \hbar$ ].

$0 \mathrm{I}$ - aye boson [inflaton]. 0.5Q - quarks. 0.5R - arcs. 1J - jay boson [Pauli repulsion]. $1 \mathrm{U}$ - gluons.

$\mathrm{Fr}$ - can model as free. Irlc - always models as interlaced. Fr/Irlc - Some SG components associate with Fr and some SG components associate with Irlc.

Figure 2: Known and suggested elementary particles. The following sentences introduce symbols for and notions about suggested new elementary particles. (The number $S$ in a symbol $S \Phi$, associates with elementary-particle spin in units of $\hbar$.) $0.5 \mathrm{~N}^{\prime}$ associates with three spin-one-half heavy neutrinos. $2 \mathrm{G}$ associates with a spin-two graviton. $3 \mathrm{G}$ associates with a spin-three relative of the photon and the graviton. $4 \mathrm{G}$ associates with a spin-four relative of the photon and the graviton. 0I associates with a spin-zero inflaton. $0.5 \mathrm{R}$ associates with three spin-one-half zero-charge analogs to quarks. $1 \mathrm{~J}$ associates with a spin-one zero-charge boson that associates with Pauli repulsion. 


\section{Eras regarding the formation of some galaxies}

\begin{tabular}{|c|c|c|c|c|}
\hline \multirow[b]{2}{*}{ Phenomena } & \multirow[b]{2}{*}{ Initiating force } & \multirow[b]{2}{*}{ RDF } & \multicolumn{2}{|c|}{ Copyright $\odot 2021$ Thomas J. Buckholtz } \\
\hline & & & Reach & PROP solution \\
\hline A one-isomer original clump (or, halo) forms & Attractive & $\mathrm{r}^{-4}$ & 1 & $2 g 12 ` 3$ \\
\hline The clump repels stuff that associates with one other isomer & Repulsive & $r^{-3}$ & 2 & $2 g 2 \cdot 4$ \\
\hline The clump attracts stuff that associates with five isomers & Attractive & $\mathrm{r}^{-2}$ & 6 & $2 \mathrm{~g} 2$ \\
\hline llisions result in a DM:OM ratio of $5^{+}: 1$ & Attractive & $\mathrm{r}^{-2}$ & 6 & $2 \mathrm{~g} 2$ \\
\hline
\end{tabular}

Legend:

The era that associates with a row in the table precedes eras that associate with subsequent rows in the table.

Initiating forces might tend to gain prominence before - and dominate for at least early parts of - the respective eras.

RDF - radial dependence of force [ $r$ denotes the distance between two significantly massive objects.].

Reach (for one instance of the initiating force) - number of isomers.

Some galaxies do not evolve beyond the first era. Some of these galaxies have essentially only ordinary matter. Some have essentially only dark matter.

During the second era, the repulsive force component also repels some stuff that associates with the isomer that associates with the original clump.

Based on the repulsive force, some galaxies have nearby stuff that associates essentially with only five isomers.

Some galaxies do not evolve beyond the third era. A DM:OM ratio of $\sim 4: 1$ can pertain. \{DM - dark matter. OM - ordinary matter\}

Collisions can merge stuff from galaxies for which the original-clump isomers differ.

Some galaxies might associate with more than one original clump and with more than one original-clump isomer.

Figure 5: Suggested eras and suggested DM:OM ratios for galaxies. For each row, the leftmost column describes aspects of the era. The rightmost four columns associate with a noteworthy cause for the era. The noteworthy cause might gain prominence before the era starts. Some galaxies do not transit beyond some eras. Our work points to possible propensities for nature to form galaxies with DM:OM ratios of approximately one to zero-plus (that is, dark matter galaxies), five-plus to one, four to one, and zero-plus to one. Galaxies that both had more than one original clump and had three original-clump isomers might tend to cease star formation earlier than do some other galaxies.

\section{Possible relationships between physics properties}

(Based on properties of some elementary particles)

Copyright $@ 2021$ Thomas J. Buckholtz

Possible relationship - for elementary bosons - between the properties of mass, spin, and charge

$(\text { Mass })^{2}=\left(\left(\text { Mass }_{\text {Higgs }}\right)^{2} / 17\right) \times\left(j^{2}+l_{m}-S^{2}-Q(Q+1)\right)$

Possible relationship between electromagnetism and gravity (interrelates one charge and two masses)

Define $\beta:(4 / 3) \times\left(\beta^{2}\right)^{6} \equiv\left(\left(\mathrm{q}_{\mathrm{e}}\right)^{2} /\left(4 \pi \varepsilon_{0}\right)\right) /\left(\mathrm{G}_{\mathrm{N}}\left(\mathrm{m}_{\mathrm{e}}\right)^{2}\right)$

Define $\beta^{\prime}: \beta^{\prime} \equiv \mathrm{m}_{\text {tau }} / \mathrm{m}_{\mathrm{e}}$

Posit: $\beta^{\prime}=\beta$

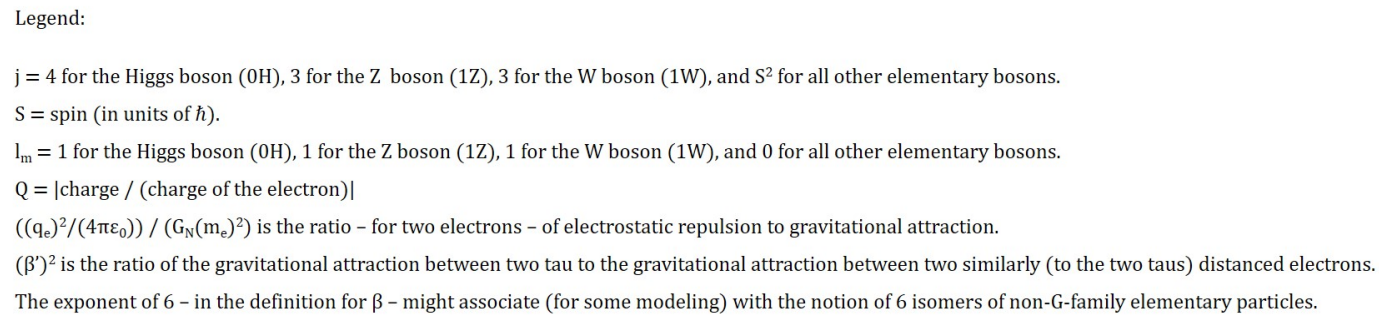

Figure 6: Possible relationships between physics properties. The boson-centric relationship might pertain for all known elementary bosons and for all new bosons that we suggest. The possible relationship between electromagnetism and gravity enables computing a tau mass that is compatible with experimental results. 


\section{Rest energies for elementary fermions}

\begin{tabular}{llcll}
\hline S $\Phi$ & Elementary particle & Approximate rest energy & Note \\
\hline $0.5 \mathrm{C}$ & Electron & $0.5109989 \ldots$ & $\mathrm{MeV}$ & Exp \\
$0.5 \mathrm{C}$ & Muon & $105.658 \ldots$ & $\mathrm{MeV}$ & Exp \\
$0.5 \mathrm{C}$ & Tau & $1776.8400 \pm 0.0115$ & $\mathrm{MeV}$ & Calc
\end{tabular}

\begin{tabular}{|c|c|c|}
\hline$S \Phi$ & Elementary particle & Approximate rest energy \\
\hline $0.5 \mathrm{~N}$ & Neutrinos - two or three & $3.4 \times 10^{-2} \quad \mathrm{eV}$ \\
\hline $0.5 \mathrm{~N}$ & Neutrinos - one or zero & $\leq 4.4 \times 10^{-4} \quad \mathrm{eV}$ \\
\hline
\end{tabular}

\begin{tabular}{ll|rl|}
\hline $0.5 \mathrm{Q}$ & Up (quark) & 2.2 & $\mathrm{MeV}$ \\
$0.5 \mathrm{Q}$ & Down (quark) & 4.8 & $\mathrm{MeV}$ \\
$0.5 \mathrm{Q}$ & Charm (quark) & $1.27 \times 10^{3}$ & $\mathrm{MeV}$ \\
$0.5 \mathrm{Q}$ & Strange (quark) & $9.3 \times 10^{1}$ & $\mathrm{MeV}$ \\
$0.5 \mathrm{Q}$ & Top (quark) & $1.71 \times 10^{5}$ & $\mathrm{MeV}$ \\
$0.5 \mathrm{Q}$ & Bottom (quark) & $4.18 \times 10^{3}$ & $\mathrm{MeV}$
\end{tabular}

$\begin{array}{llrl}0.5 \mathrm{R} & \text { Arc } & 8.6 \text { to } 10.7 & \mathrm{MeV} \\ 0.5 \mathrm{R} & \text { Arc } & 6.8 \text { to } 8.6 & \mathrm{MeV} \\ 0.5 \mathrm{R} & \text { Arc } & 102 \text { to } 106 & \mathrm{MeV} \\ 0.5 \mathrm{~N}^{\prime} & \text { Heavy neutrino } & \geq 6 \times 10^{3} & \mathrm{GeV} \\ 0.5 \mathrm{~N}^{\prime} & \text { Heavy neutrino } & \geq 6 \times 10^{3} & \mathrm{GeV} \\ 0.5 \mathrm{~N}^{\prime} & \text { Heavy neutrino } & \geq 6 \times 10^{3} & \mathrm{GeV}\end{array}$

Legend:

Figure 7: Possible rest energies for elementary fermions. Eight standard deviations of calculated tau rest energy fit within one standard deviation of experimental tau rest energy. The calculated quark masses comport with experimental results. Results regarding neutrinos comport with the notion of three (not heavy) neutrinos and with astrophysics data. Our work suggests possible ranges for the masses of each of the three possible arc elementary fermions.

its CURR partner can adequately associate with special relativity.

Our LRI modeling for electromagnetism has some similarities to modeling based on charge-andcurrent 4-vectors and has some parallels to modeling based on an electric field, a magnetic field, and Maxwell's equations. However, some differences pertain. For example, LRI modeling includes a PROP solution that associates with a component of magnetic field that associates with the notion of charge dipoles. That PROP solution has a CURR partner that associates with a current of charge dipoles.

Our LRI modeling for gravitation has similarities and differences with respect to gravitoelectromagnetism. (Regarding gravitoelectromagnetism, see references [1] and [2].)

SRI solutions and ELF solutions come in PROP and CURR pairs. Each known SRI particle associates with a solution pair. Each not-yet-found SRI particle that this essay suggests associates with a solution pair. Each known ELF particle associates with a solution pair. Each not-yet-found ELF particle that this essay suggests associates with a solution pair.

We think that modeling based on the LRI, SRI, and ELF aspects discussed above suffices to point to possibly relevant new physics. For example, we interpret our modeling as describing aspects of two possible eras - in the evolution of the universe - before the possible inflationary epoch. And, we interpret our modeling as suggesting a mechanism that leads to the recent multi-billion-years era increases in the rate of expansion of the universe.

However, modeling just based on LRI, SRI, and ELF aspects discussed above does not suffice to explain some data about ratios of dark matter to ordinary matter and does not suffice to explain the magnitude of the recent multi-billion-years era increases in the rate of expansion of the universe. (Regarding ratios of dark matter to ordinary matter, perhaps preview table 7 . Regarding the magnitude of the recent multi-billion-years era increases in the rate of expansion of the universe, perhaps preview the notion of reach - or $\rho_{I}$ - in table 15.)

To explain some data about ratios of dark matter to ordinary matter, we posit that nature includes six isomers of the set of SRI and ELF elementary particles. Ordinary matter associates with all of the SRI particles in so-called ELPI0 and all of the known ELF particles in ELPIO. The symbol ELPI denotes the three-word phrase elementary particle isomer. The integers $l$ that associate with symbols of the form ELPI $l$ range from zero to five. Dark matter associates with some yet-to-be-found ELF particles in EPLI0 and with all elementary particles in ELPI1 through ELPI5.

The symbol STUI denotes stuff - such as hadron-like particles, atoms, and stars - made up of just (or essentially just) ELPI $l$ elementary particles (plus LRI aspects that include electromagnetism and gravity).

We posit that, across isomers, the ELPI have similarities. We posit that the mass of each SRI particle in any one isomer is the same as the mass of a counterpart SRI elementary particle in each 
other isomer. We posit that the mass of each ELF particle in any one isomer is the same as the mass of a counterpart ELF elementary particle in each other isomer.

We posit that the six ELPI differ in at least one way. For each of isomer-zero and isomer-three, the flavour of the lowest-mass charged lepton equals the flavour for the two lowest-mass quarks. For each of the other four isomers, the flavour of the lowestmass charged lepton does not equal the flavour of the two lowest-mass quarks. (Perhaps, preview table 14.) One possible other difference between isomers might be that ELPI0, ELPI2, and ELPI4 associate with left-handedness (for, for example, charged leptons) and ELPI1, ELPI3, and ELPI5 associate with right-handedness.

Regarding LRI, a so-called reach associates with each PROP-and-CURR pair. We use the symbol $\rho_{I}$ to denote reach. Allowed values for $\rho_{I}$ are one, two, and six. For relatively familiar physics such as the physics of solar systems - the dominant gravitational PROP-and-CURR pair has a reach of six. The six STUI interact with each other via this component of gravity. Regarding electromagnetism, the reach that associates with the chargeand-charge-current PROP-and-CURR pair is one. The reach that associates with the charge-dipoleand-related-current PROP-and-CURR pair is one. Each one of the STUI has, in effect, its own instance of each of these two electromagnetic-centric PROP-and-CURR pairs. Each STUI does not interact with any other STUI via either of these two electromagnetic-centric PROP-and-CURR pairs.

The notion of six isomers and the notion of instances of LRI PROP-and-CURR pairs seem to suffice to explain ratios of dark matter to ordinary matter. (Perhaps, preview table 7.) The two notions might suffice to explain the size of the recent multi-billion-years era increases in the rate of expansion of the universe. (Perhaps, preview table 15.)

\subsection{Relationships between our work and other work}

We discuss relationships between our work and other work. Here, other work includes observational research and modeling-centric research.

\subsubsection{We discuss relationships - between our work and other work - regarding elementary parti- cles, physics constants, and physics proper- ties.}

We discuss other work that tries to suggest new elementary particles.

Reference [3] lists some types of modeling that people have considered regarding trying to extend the elementary particle Standard Model, including trying to suggest elementary particles that people have yet to find. Types of models associate with terms such as large extra dimensions, Kaluza-Klein (which associates with notions of gravity in more than four dimensions), grand unification, supersymmetry, and superstrings. Reference 4 provides information about some of these types of modeling. References [5], 66, and [7] provide some information about modeling and about experimental results. Reference [8] provides other information about modeling and about experimental results. (Perhaps, see reviews numbered 86, 87, 88, 89, 90, and 94.)

We discuss possible elementary particles that people have yet to find and we suggest.

Reference [9] suggests the notions of dark matter charges and dark matter photons. We suggest dark matter isomers of charged elementary particles and, in effect, dark matter components - such as components associating with electrostatics and magnetostatics - of electromagnetism.

Reference [10] suggests the notion of a so-called inflaton field. We suggest an inflaton elementary particle. (Perhaps, preview table 9 and note the 0I boson.)

People suggest the notion of a graviton. (See, for example, reference [11.) We suggest a graviton. (Perhaps, preview table 9.)

Reference 12 discusses notions of sterile neutrinos and heavy neutrinos. We suggest possible elementary particles that might associate with notions of heavy neutrinos. (Perhaps, preview table 9.)

We discuss possible elementary particles that people have yet to find and our modeling seems not to suggest.

Reference [7] reviews modeling and experiments regarding so-called magnetic monopoles. Reference [7] notes that a symmetry regarding Maxwell's equations suggests that nature might include magnetic monopoles. We suggest that nature might not include an interaction that would associate with magnetic monopoles. (Perhaps, preview table 3 .)

Reference [5] reviews modeling and experiments regarding so-called axions. Reference [5] notes modeling that suggests that nature might include axions. We suggest that nature might not include axions. (Perhaps, preview table 5.) We suggest that phenomena that people might attribute to axions might not associate with axions. One such phenomenon could be electromagnetic interactions between ordinary matter and dark matter based on, for example, the so-called $1 \mathrm{~g} 1^{\prime} 2^{\prime} 4$ component of electromagnetism. (Perhaps, preview table 3 and table 8.)

Reference [6] reviews modeling and experiments regarding so-called leptoquarks. We suggest that nature might not include leptoquarks. (Perhaps, preview table 5.)

We discuss prospectively some aspects, assum- 
ing that our work gains attention.

We discuss neutrino masses and oscillations.

Reference [12] discusses modeling and data about neutrino masses and oscillations.

We suggest neutrino masses. (Perhaps, preview table 13.) We also suggest that, in effect, gravity measures neutrino masses and a spin-three analog (to electromagnetism and gravity) measures neutrino flavours. (Perhaps, preview table 13.) As far as we know, our modeling is not incompatible with data that reference [12] discusses. Future experimentation might help validate or refute aspects of our work regarding neutrinos.

We discuss gravitation.

Reference [13] discusses experimental tests of theories of gravity.

We suggest effects - associating with isomers of elementary particles and with reaches of components of gravity - that suggest that other modeling regarding gravity would not be adequately accurate for some circumstances. (Perhaps, preview table 19.) This essay discusses some such circumstances. We are uncertain as to the extent to which aspects that reference [13] or reference [14] discuss would tend to validate or refute aspects of our modeling that pertains to gravitation.

We discuss physics constants and properties.

Our work seems to interrelate some physics constants. (Perhaps, preview table 10 and table 12.) Our work seems to interrelate some properties, including via modeling that catalogs physics properties. (Perhaps, preview table 3.)

We might offer new approaches to estimating some physics properties. This essay points to masses - that would comport with recent experimental results and that would have smaller standard deviations than standard deviations that associate with recent experiments - for each of the tau elementary fermion and the Higgs boson. (Perhaps, preview respectively table 12 and table 10.) This essay notes - regarding the anomalous magnetic dipole moment of the tau elementary fermion a possible estimate that might approximate a Standard Model estimate. (Perhaps, preview discussion related to table 19.) This essay notes - regarding the fraction of top quark decays that result in righthanded $\mathrm{W}$ bosons - a possible estimate that might approximate a Standard Model estimate.

\subsubsection{We discuss relationships - between our work and other work - regarding cosmology.}

We think that - with some exceptions - our work does not necessarily suggest significant changes - to concordance cosmology - regarding the large-scale evolution of the universe. (References [15], [16, and [17] review aspects of concordance cosmology.)

Each exception associates either with a possible aspect of nature for which people have no obser- vations or with a known gap between observations and concordance cosmology.

One exception pertains regarding before inflation. One exception pertains regarding recent changes in the rate of expansion of the universe. In each case, we suggest noteworthy contributions by a gravitational force component for which each instance (of the component) has a reach that is greater than one isomer. (Perhaps, preview table 8.) For times associating with between the two cases, we suggest dominance by gravitational force components that have reaches of one isomer. For times associating with between the two cases, we do not propose significant incompatibilities between our work and large-scale concordance cosmology.

We discuss a possibility regarding times before inflation. (Reference [16] discusses inflation.)

We think that no direct observations pertain. We suggest two eras before inflation. (Perhaps, preview table 15.) The first of those two eras features aspects that the Standard Model and concordance cosmology do not include. One aspect is the so-called jay boson. (Perhaps, preview table 9 and table 15.) The other aspect is the socalled $2 \mathrm{~g} 1^{\prime} 2^{6} 3^{6} 8^{6} 16$ component of gravity. (Perhaps, preview table 15.) An instance of that component has a reach of six isomers. For purposes of discussion, we assume that the universe transited those two eras. We assume that concordance cosmology can embrace the jay boson. For the first of those two eras, an extrapolation of concordance cosmology techniques might underestimate the strength of the key driver - the $2 \mathrm{~g} 1^{\prime} 2^{`} 3^{6} 8^{`} 16$ component of gravity - by a factor of six.

We discuss phenomena during and after the lead-up to the current multi-billion-years era of increases in the rate of expansion of the universe.

Various people suggest that concordance cosmology underestimates increases in the rate of expansion. (References [17], [18, [19], [20], and [21] discuss relevant notions.)

We think that we point to a basis for the underestimates. Regarding times before that lead-up, we suggest dominance by instances of an attractive quadrupole gravitational force component (that is, $2 \mathrm{~g} 1^{6} 2^{6} 3$ ) with a reach of one isomer. (Perhaps, preview table 15.) Before and during the recent multibillion-years era, the $2 \mathrm{~g} 2{ }^{\prime} 4$ gravitational force component gains prominence and then becomes dominant. Each instance of $2 \mathrm{~g} 2^{6} 4$ has a reach of two isomers. We suggest that concordance cosmology models that work well regarding times for which reach-one dominance pertains would not necessarily work well after those times. We suggest that extrapolating based on such concordance cosmology modeling would underestimate (conceptually by a factor of two) the strength of the driver for increases in the rate of expansion. We suggest that - to get good re- 
sults via concordance cosmology modeling - people might adjust the equation of state. In general, for each relevant density, components of pressure that associate with repulsion need to increase.

Our suggested resolution regarding the underestimate seems to differ considerably from possible resolutions based on concordance cosmology modeling. Our suggested resolution focuses on phenomena that would pertain at the times for which concordance cosmology modeling seems not to be adequate. Other possible resolutions seem to focus on phenomena early in the history of the universe. (See reference [17].)

\subsubsection{We discuss relationships - between our work and other work - regarding astrophysics.}

We think that our modeling is not necessarily incompatible with astrophysics data or with results based on concordance cosmology modeling. (Here, we assume that the two-word term concordance cosmology includes aspects that associate with dark matter, astrophysics, and effects of gravity on scales as small as one galaxy.)

We discuss properties of dark matter.

Reference 22] suggests the following notions. Most dark matter comports with notions of cold dark matter. Models that associate with the twoword term modified gravity might pertain; but - to the extent that the models suggest long-range astrophysical effects - such models might prove problematic. People suggest limits on the masses of basic dark matter objects. Observations suggest socalled small-scale challenges to the notion that all dark matter might be cold dark matter. People use laboratory techniques to try to detect dark matter. People use astrophysical techniques to try to infer properties of dark matter.

We think that our modeling regarding dark matter comports with such notions. For astrophysical phenomena (and not necessarily regarding the rate of expansion of the universe), components - that have reaches other than six - of gravity play roles locally; however, the impacts do not extend to cosmological scales. The dark matter isomer that might evolve similarly to ordinary matter might provide bases for resolving some of the so-called small-scale challenges.

We discuss observations and models regarding galaxy formation.

Reference 23] discusses galaxy formation and evolution, plus contexts in which galaxies form and evolve. Reference [23] discusses parameters by which people classify and describe galaxies.

We suggest that - regarding galaxies - observations of ratios of dark matter to ordinary matter might tend to cluster near some specific ratios. (Perhaps, preview table 7.) Our modeling seems to explain such ratios.
Our modeling suggests that ratios of dark matter to ordinary matter might reflect fundamental aspects - of nature - that concordance cosmology modeling does not include. Here, a key aspect is that of isomers. (Perhaps, preview table 7.)

Reference [23] seems not to preclude galaxies that have few ordinary matter stars. Reference [23] seems not to preclude galaxies that have little ordinary matter.

We think that dark matter to ordinary matter ratios that our modeling suggests are not necessarily incompatible with verified concordance cosmology modeling.

We discuss observations and models regarding interactions between galaxies.

Reference [24] suggests that concordance cosmology modeling might not adequately explain gravitational interactions between neighboring galaxies. We suggest that notions pertaining to reaches and isomers might bridge the gap between observations and concordance cosmology modeling.

We think that our work points to a possible opportunity to study harmony between results based on established kinematics models and results based on our notions of components of gravity.

\section{Methods}

This unit develops and deploys modeling that matches all known elementary particles; suggests new elementary particles; interrelates elementary particles, properties of individual objects, and properties of systems of objects; and provides specifications for dark matter.

The method that we develop here outputs solutions to equations that involve sums of integers.

Some solutions associate with modeling that has similarities to modeling based on Maxwell's equations, to modeling based on charge-and-current 4vectors, or to modeling based on the notion of gravitoelectromagnetism. (References [1] and [2] discuss gravitoelectromagnetism.) Some solutions associate with electromagnetic fields - such as an electric field or a magnetic field - and with electromagnetic properties - such as charge and magnetic dipole moment - of systems. Some solutions associate with gravitational fields and with gravitational properties - such as mass.

Some solutions point to radial spatial dependences of potentials. (Regarding radial spatial dependences of potentials and forces, we use terminology that generally associates with Newtonian kinematics.) One such radial spatial dependence of potential is $r^{-1}$ for a component of electromagnetism that associates with the charge of a system. Here, $r$ denotes a distance away from the system that produces the component of electromagnetism. Another such radial spatial dependence of potential is 
$r^{-2}$ for a component of electromagnetism that associates with the magnetic dipole moment of the system. (Generally, for radial spatial dependences other than $r^{-1}$, angular dependences also pertain.)

We associate the symbol $1 \mathrm{~g}$ with solutions that associate with electromagnetism. Here, the one denotes the spin (in units of $\hbar$ ) of photons. We associate the symbol $2 \mathrm{~g}$ with solutions that associate with gravitation. Here, the two denotes the spin (in units of $\hbar$ ) of (as yet not detected) gravitons. We associate the symbol $3 \mathrm{~g}$ with solutions that associate with a would-be spin-three elementary boson. We associate the symbol $4 \mathrm{~g}$ with solutions that associate with a would-be spin-four elementary boson. We associate the two-element term long-range force (or, the two-element term long-range interaction) with each one of $1 \mathrm{~g}$ through $4 \mathrm{~g}$.

Other solutions associate mathematically with 0g. Some $0 \mathrm{~g}$ solutions associate with elementary bosons, such as the $\mathrm{Z}$ boson and the $\mathrm{W}$ boson. Some $0 \mathrm{~g}$ solutions associate with elementary fermions, such as quarks and charged leptons.

The method outputs solutions that seem to match all known elementary particles and that suggest new elementary particles.

We use the following method to catalog elementary particles. (Perhaps, preview table 9.) A symbol of the form $S \Phi$ associates with a so-called family of elementary particles. Each elementary particle associates with one family. Each family associates with one of one, three, or eight elementary particles. For a family, the value $S$ denotes the spin (in units of $\hbar$ ) for each elementary particle in the family. $S$ associates with the expression $S(S+1) \hbar^{2}$ that associates with angular momentum. Values of $S$ include 0, 0.5, 1, 2, 3, and 4. Except regarding quarks and possible heavy neutrinos, the family associates with a one-letter value of $\Phi$. For quarks, $\Phi=Q_{1 / 3}$ associates with each quark for which the magnitude of the charge is one-third the magnitude of the charge of the electron. $\Phi=\mathrm{Q}_{2 / 3}$ associates with each quark for which the magnitude of the charge is two-thirds the magnitude of the charge of the electron. For neutrino-like particles, $\Phi=\mathrm{N}$ associates with the three known neutrinos. $\Phi=\mathrm{N}$ ' associates with possible particles for which we use the two-word term heavy neutrinos.

\subsection{Charge, mass, other properties, and long-range forces}

This unit develops and deploys modeling that interrelates elementary particles (such as the photon) that carry long-range forces, properties of individual objects, and properties of systems that include individual objects.

\subsubsection{We explore notions that point toward aspects of some modeling that our work uses.}

We imagine two non-moving objects - object A and object $\mathrm{B}$ - that are located a distance $r$ from each other. Each object has non-zero charge and non-zero mass. We consider the impacts of fields such as electromagnetism or gravity - generated by object $\mathrm{A}$ on object B.

The electric potential that affects object $B$ varies as $r^{-1}$. The gravitational potential that affects object $\mathrm{B}$ varies as $r^{-1}$.

We imagine hypothetical effects that associate with hypothetical interactions by object $\mathrm{B}$ with a hypothetical combination - produced by object A of electric field and gravitational field. We imagine that the potential that associates with these interactions varies as $r^{-1}$ times $r^{-1}$, which equals $r^{-2}$.

We discuss aspects of hypothetical particles that might intermediate interactions between object $\mathrm{A}$ and object $\mathrm{B}$. We use the two-item term object $\mathrm{C}$ to denote such a hypothetical particle.

We imagine objects $\mathrm{C}$ that have some similarities to and some differences from either an atom or a solar system. One or more components of an object $\mathrm{C}$ orbit a point that is central to object $\mathrm{C}$. An object $\mathrm{C}$ exhibits orbitals. Each orbital associates with a unique magnitude $l_{o} \hbar$ of orbital angular momentum. Here, $l_{o}$ is a positive integer. Up to one entity can associate with (or, occupy) an orbital. The integer $l_{\max }$ denotes the maximum value of $l_{0}$ that associates with an occupied orbital. Relative to an axis that runs from object $\mathrm{A}$ to object $\mathrm{B}$, the angular momentum that associates with an occupied orbital is one of $-l_{o} \hbar$ and $+l_{o} \hbar$. (We exclude - for the occupied orbital that associates with $l_{o}$ values of $l$ for which $-l_{o}<l<+l_{o}$.) The angular momentum that associates with an unoccupied orbital is $0 \hbar$. (Regarding considering the object to be atom-like, the following notions pertain. The nucleus has zero spin. Entities that occupy orbitals have zero spin. Entities that occupy orbitals do not interact with each other.) Relative to the axis, the total angular momentum that associates with an object $\mathrm{C}$ is the sum - over the occupied orbitals - of the respective $\pm l_{o} \hbar$.

Regarding modeling that we discuss below, the following notions pertain.

- Individual objects $\mathrm{C}$ do not associate directly with elementary particles.

- Mathematical modeling regarding an object C associates with a so-called (mathematical) solution. Some sets of solutions associate with modeling for LRI (or, long-range interaction) elementary particles such as the photon. Some sets of solutions associate with modeling for SRI (or, short-range interaction) elementary bosons such as the $\mathrm{W}$ boson. Some sets 
of solutions associate with modeling for ELF (or, elementary fermion) elementary fermions such as the electron.

- Spatial dependences of potentials can depend on angular coordinates as well as on a radial coordinate such as $r$.

\subsubsection{We develop modeling that associates aspects of long-range forces with properties of objects that interact via long-range forces.}

We focus on mathematics that associates with the discussion about objects $\mathrm{C}$ and orbitals. We do not explore the notion of direct physics relevance for such objects. We do not explore the notion of such objects. For convenience, we continue to use the word orbital.

The discussion about hypothetical objects $\mathrm{C}$ suggests expressions of the form $\sum_{o \in O}\left( \pm l_{o}\right)$. Here, $O$ denotes the set of occupied orbitals. $o$ denotes a member of $O$.

We define $\Sigma$ to be the absolute value of the sum of the various values of $\pm l_{o}$. The equation $\Sigma=\left|\sum_{o \in O}\left( \pm l_{o}\right)\right|$ pertains. The term two-word Diophantine equations associates with the modeling that we pursue.

We define $\Gamma$ to be the list of relevant values of $l_{o}$. We define $n_{\Gamma}$ to be the number of elements in the list $\Gamma$. The symbol $l_{\max }$ denotes the maximum value of $l_{o}$ in $\Gamma$. It follows that $n_{\Gamma}$ does not exceed $l_{\max }$. We use the symbol $\Sigma \mathrm{g} \Gamma$ to denote the combination of a list $\Gamma$ and a relevant value of $\Sigma$. The letter $\mathrm{g}$ anticipates an association with electromagnetism and an association with gravity. (Perhaps, think of $\mathrm{g}$ as in gamma rays and $\mathrm{g}$ as in gravity.) Within a list $\Gamma$, we separate values of $l_{o}$ by using the symbol '. For example, for the $\Gamma$ symbolized by $1^{\prime} 2,1 \in \Gamma$ and $2 \in \Gamma$.

Table 1 alludes to all $\Sigma \mathrm{g} \Gamma$ solutions, for which $1 \leq l_{o} \leq l_{\max } \leq 4$ and no two values of $l_{o}$ are the same.

We associate the symbol $\Sigma \mathrm{g}$ with solutions of the form $\Sigma \mathrm{g} \Gamma$. We associate the symbol $\Sigma \mathrm{g}$ ' with $\Sigma$ g solutions for which $\Sigma \in \Gamma$. We associate the symbol $\Sigma$ g" with $\Sigma$ g solutions for which $\Sigma \notin \Gamma$.

We explore the notion that solutions that table 1 lists associate with long-range interactions (or, LRI) and with properties - of physical objects such as planets or elementary particles - that people do infer or might infer via observations based on information carried by electromagnetic fields, gravitational fields, and possibly other similar fields.

Regarding observations - via electromagnetism - pertaining to an object with nonzero charge, people might infer both a magnitude of charge of the object and a velocity with which the object moves. For models based on special relativity, the notion of a charge-and-charge-current 4-vector pertains.
We deploy the symbol PROP to associate with $\sum g \Gamma$ solutions that associate with properties. We deploy the symbol CURR to associate with $\mathrm{\Sigma g} \Gamma$ solutions that associate with currents of properties.

We extend the notions of PROP and CURR to apply widely regarding modeling regarding LRI. We anticipate that, for each LRI PROP solution there is an LRI CURR solution.

We posit the following associations. $1 \mathrm{~g}$ associates with electromagnetism. $2 \mathrm{~g}$ associates with gravitation. $3 \mathrm{~g}$ associates with interactions with a function of elementary fermion flavor. $4 \mathrm{~g}$ associates with interactions with magnitude of internal angular momentum. $l_{o}=4$ associates with rotation. (For a preview regarding the usefulness of these posits, see the $\Sigma$ column and the properties column in table 3 .)

We posit that a solution associates with a socalled RDP of the form $\Xi^{-n_{\Sigma \mathrm{g} \Gamma}}$. RDP stands for radial dependence of potential. Here, we consider Newtonian modeling for potentials (as in potential energy) that associate with fields (such as the electromagnetic field and the gravitational field) that an object produces. For a solution other than a monopole solution, the potential can (and generally does) vary based on angular coordinates (as well as based on a radial coordinate). We posit that $\Xi^{-1}=r^{-1}$, in which $r$ is the spatial distance from the object.

We note, but do not comment further regarding, the notions that the posited RDP of the form $\Xi^{-1}$ associates with a physics use of the word monopole and that mentions in table 1 of the word monopole associate with a mathematics use of the word monopole. Similar parallel notions pertain regarding each of the words dipole, quadrupole, and so forth. (Also, we provide a cautionary note regarding terminology. Per table 2, we associate the solution for which $\Sigma$ is one and $\Gamma$ is $1^{6} 2^{6} 4$ with each one of the following: $\Xi^{-3}$ and hence mathematical quadrupole, $r^{-3}$ and hence a behavior of potential that associates with a notion of quadrupole, and a physics object that associates with a magnetic dipole that rotates around an axis that does not equal the axis that associates with the magnetic dipole. One way to think about the seeming tension between quadrupole and dipole is to associate the factor $\Xi^{-1}$ that associates with $l_{o}=4$ with $(c t)^{-1}$ instead of with $r^{-1}$. Here, $c$ denotes the speed of light and $t$ denotes the time that light takes to go from the magnetic-dipole object to the distance $r$ from the object. This interpretation has consistency with the notion that the relevant quadrupole component of the electromagnetic field associates with an object that people might characterize as having the properties of a magnetic dipole.)

For the moment, we ignore solutions for which $\Sigma=0$ and solutions for which $\Sigma \geq 5$. 
Table 1: $\Sigma \mathrm{g} \Gamma$ solutions, assuming that $1 \leq l_{o} \leq l_{\max } \leq 4$ and that no two values of $l_{o}$ are the same. The symbol 0 is a placeholder for an unused pair, $-l_{o}$ and $+l_{o}$, of values. For each row, there are $2^{n_{\Gamma}}$ possible ways to assign signs regarding the set of $n_{\Gamma}$ terms. There are $2^{n_{\Gamma}}$ expressions of the form $\sum_{o \in O}\left( \pm l_{o}\right)$. Thus, there are $2^{n_{\Gamma}-1}$ solutions for $\Sigma=\left|\sum_{o \in O}\left( \pm l_{o}\right)\right|$. The number $n_{\Sigma \mathrm{g} \Gamma}$ equals $2^{n_{\Gamma}-1}$ and states the number of solutions. For example, for $l_{\max }=2$ and $n_{\Gamma}=2$, the solutions are $1 \mathrm{~g} 1^{\prime} 2$ (as in $\left.1=|-1+2|\right)$ and $3 \mathrm{~g} 1^{\prime} 2$ (as in $3=|+1+2|$ ). The notion column refers to the number of solutions. For monopole, one solution pertains. For dipole, two solutions pertain. For quadrupole, four solutions pertain. For octupole, eight solutions pertain. The $\Sigma$ column shows values of $\Sigma$ that associate with solutions. For the case of octupole, each one of $\Sigma=2$ and $\Sigma=4$ associates with two solutions. Regarding $\Sigma=2,|-1+2-3+4|=2=|-1-2-3+4|$. Regarding $\Sigma=4,|-1-2+3+4|=4=|+1+2-3+4|$. The symbol $n_{o 0}$ denotes the number of times the symbol 0 associates with an $l_{o}$ for which $1 \leq l_{o} \leq l_{\max }$.

\begin{tabular}{|c|c|c|c|c|c|c|c|c|c|c|}
\hline$l_{\max }$ & $n_{\Gamma}$ & $l_{o}=1$ & $l_{o}=2$ & $l_{o}=3$ & $l_{o}=4$ & $\Gamma$ & $n_{\Sigma \mathrm{g} \Gamma}$ & Notion & $\Sigma$ & $n_{o 0}$ \\
\hline 1 & 1 & \pm 1 & - & - & - & 1 & 1 & Monopole & 1 & 0 \\
\hline 2 & 1 & 0 & \pm 2 & - & - & 2 & 1 & Monopole & 2 & 1 \\
\hline 2 & 2 & \pm 1 & \pm 2 & - & - & $1^{‘} 2$ & 2 & Dipole & 1,3 & 0 \\
\hline 3 & 1 & 0 & 0 & \pm 3 & - & 3 & 1 & Monopole & 3 & 2 \\
\hline 3 & 2 & \pm 1 & 0 & \pm 3 & - & $1^{\prime} 3$ & 2 & Dipole & 2,4 & 1 \\
\hline 3 & 2 & 0 & \pm 2 & \pm 3 & - & $2 \cdot 3$ & 2 & Dipole & 1,5 & 1 \\
\hline 3 & 3 & \pm 1 & \pm 2 & \pm 3 & - & $1^{‘} 2^{\prime} 3$ & 4 & Quadrupole & $0,2,4,6$ & 0 \\
\hline 4 & 1 & 0 & 0 & 0 & \pm 4 & 4 & 1 & Monopole & 4 & 3 \\
\hline 4 & 2 & \pm 1 & 0 & 0 & \pm 4 & $1^{‘} 4$ & 2 & Dipole & 3,5 & 2 \\
\hline 4 & 2 & 0 & \pm 2 & 0 & \pm 4 & $2^{\circ} 4$ & 2 & Dipole & 2,6 & 2 \\
\hline 4 & 2 & 0 & 0 & \pm 3 & \pm 4 & $3^{6} 4$ & 2 & Dipole & 1,7 & 2 \\
\hline 4 & 3 & \pm 1 & \pm 2 & 0 & \pm 4 & $1^{6} 2^{6} 4$ & 4 & Quadrupole & $1,3,5,7$ & 1 \\
\hline 4 & 3 & \pm 1 & 0 & \pm 3 & \pm 4 & $1^{6} 3^{6} 4$ & 4 & Quadrupole & $0,2,6,8$ & 1 \\
\hline 4 & 3 & 0 & \pm 2 & \pm 3 & \pm 4 & $2^{\prime} 3^{‘} 4$ & 4 & Quadrupole & $1,3,5,9$ & 1 \\
\hline 4 & 4 & \pm 1 & \pm 2 & \pm 3 & \pm 4 & $1^{6} 2^{\circ} 3^{6} 4$ & 8 & Octupole & $0,2,2,4,4,6,8,10$ & 0 \\
\hline
\end{tabular}

Table 2: PROP $\Sigma \mathrm{g}$ ' solutions, for $l_{\max } \leq 4$. Here, the allowed values of $l_{o}$ are $1,2,3$, and 4 . The table suggests possible contributions - from one object - to each of four $\Sigma \mathrm{g}$. $1 \mathrm{~g} 1$ associates with a component - of the electromagnetic field that the object produces - that associates with the object's charge. The word scalar associates with this solution. 1g1'2 associates with the object's magnetic field. An axis associates with that field. The one-element term 3-vector associates with this solution. 1g1'2'4 associates with a combination of magnetic field and rotation (over time) of the axis of the magnetic field. (The Earth is an object for which the axis of rotation does not equal the axis of the magnetic field.) $2 \mathrm{~g} 2$ associates with the object's mass. The word scalar associates with this solution. $2 \mathrm{~g}^{\prime} 4$ associates with rotation of the object's mass. An axis associates with that rotation. The one-element term 3-vector associates with this solution. (Regarding general relativity, this solution seems to associate with aspects of rotational frame dragging.) $2 \mathrm{~g} 1^{\prime}{ }^{\prime}{ }^{\prime} 3$ associates with a non-spherically symmetric distribution of mass. $2 \mathrm{~g} 1^{\prime} 2^{\prime} 3^{6} 4 \mathrm{v}$ associates with rotation (of a non-spherically symmetric distribution of mass)

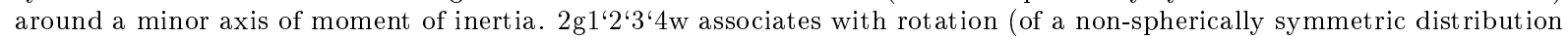
of mass) around a major axis of moment of inertia. For gravity produced by an object like the Sun, PROP 2g' solutions other than $2 \mathrm{~g} 2$ associate with adjustments with respect to the gravity that associates with $2 \mathrm{~g} 2$. Regarding large-scale gravitation, PROP 2g' solutions other than $2 \mathrm{~g} 2$ can associate with gravitational effects that dominate gravitational effects that associate with $2 \mathrm{~g} 2$. We postpone discussing $3 \mathrm{~g}$ ' solutions and $4 \mathrm{~g}$ ' solutions.

\begin{tabular}{|c|c|c|c|c|}
\hline$\Sigma$ & Monopole & Dipole & Quadrupole & Octupole \\
\hline 1 & $1 \mathrm{~g} 1$ & $181^{\circ} 2$ & $1 g 1^{6} 2^{\circ} 4$ & - \\
\hline 2 & $2 \mathrm{~g} 2$ & $2 \mathrm{~g} 2^{\prime} 4$ & $2 \mathrm{~g} 1^{‘} 2^{‘} 3$ & 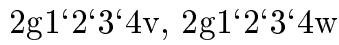 \\
\hline 3 & $3 \mathrm{~g} 3$ & - & $3 g 2^{6} 3^{\circ} 4$ & - \\
\hline 4 & $4 \mathrm{~g} 4$ & - & - & $4 \mathrm{~g} 1^{\circ} 2^{\circ} 3^{6} 4 \mathrm{v}, 4 \mathrm{~g} 1^{\circ} 2^{\circ} 3^{\circ} 4 \mathrm{w}$ \\
\hline
\end{tabular}


Table 2 explores the notion that solutions have meaning regarding physics.

We suggest that table 2 points to a useful basis for modeling regarding, at least, electromagnetism and gravity.

Notions of three degrees of freedom seem to pertain regarding solutions that table 2 shows.

The following examples - of three degrees of freedom - pertain regarding 1g' solutions. Regarding $\lg 1^{`} 2$, three degrees of freedom pertain. Two degrees of freedom associate with the orientation of the magnetic moment 3 -vector. One degree of freedom associates with the magnitude of the magnetic moment 3 -vector. Compared to $1 \mathrm{~g} 1^{`} 2$, $1 \mathrm{~g} 1^{`} 2^{\circ} 4$ has three more degrees of freedom. Two degrees of freedom associate with the orientation of the angular momentum 3-vector. One degree of freedom associates with the magnitude of the angular momentum 3-vector.

Regarding each of the solutions that table 2 shows, $l_{o}=4$ seems to associate - regarding rotation - with three degrees of freedom. However, we are careful to note that - for each of the $3 \mathrm{~g} 2^{\circ} 3^{6} 4$, $4 \mathrm{~g} 1^{6} 2^{6} 3^{6} 4 \mathrm{v}$, and $4 \mathrm{~g} 1^{6} 2^{6} 3^{6} 4 \mathrm{w}$ solutions - there is no associated same- $\Sigma \Sigma$ g' other solution to which one can add $l_{o}=4$ to obtain the subject solution.

We suggest that - for some aspects of our modeling - three degrees of freedom, mathematics associating with two one-dimensional harmonic oscillators, and mathematics associating with the group $S U(2)$ associate with each other. (For integers $l$ such that $l \geq 2$, reference 25 interrelates mathematics associating with $l$ one-dimensional harmonic oscillators and mathematics associating with the group $S U(l)$.) Here, we consider that one oscillator might associate with boson-like excitations regarding a relevant aspect that associates with the relevant value of $-l_{o}$. The other oscillator might associate with boson-like excitations regarding a relevant aspect that associates with the relevant value of $+l_{o}$. The number of generators of the group $S U(2)$ is three.

We explore notions of CURR solutions and notions of $l_{o} \geq 5$.

Regarding PROP solutions, the following notions pertain. The combination of $\Sigma=1$ and $l_{o}=1$ associates with charge. The combination of $\Sigma=2$ and $l_{o}=2$ associates with mass. The combination of $\Sigma=4$ and $l_{o}=4$ associates with angular momentum.

To explore CURR solutions, we want to add three degrees of freedom that associate with the CURR aspects of PROP-and-CURR 4-vectors.

Regarding CURR solutions that associate with PROP modeling for which $l_{\max } \leq 4$, we posit the following. $8 \in \Gamma$ associates with momentum. $l_{o}=7$ associates (as does $l_{o}=1$ for PROP modeling) with charge. $l_{o}=6$ associates (as does $l_{o}=2$ for PROP modeling) with mass. $l_{o}=5$ associates (as does $l_{o}=3$ for PROP modeling) with a function of elementary fermion flavour.

For PROP solutions we allow $l_{o}=16 \in \Gamma$. For CURR solutions we allow $l_{o}=16 \in \Gamma$ and $l_{o}=32 \in \Gamma$.

Table 3 extends table 2 and lists PROP and CURR $\Sigma$ g' solutions, for which $l_{\max } \leq 16$ for PROP $\Sigma$ '' solutions.

Table 4 lists some $\Sigma$ g" solutions for which we posit relevance. Table 4 previews the notion that $\lg 3^{6} 4$ associates with - at least - depletion (via interactions with hydrogen-like atoms) of CMB (or, cosmic microwave background radiation) by ordinary matter and some dark matter. (Perhaps, preview table 7.) Table 4 previews the notion that $3 \mathrm{~g} 1^{\prime} 2$ associates with modeling regarding anomalous magnetic moments. (Perhaps, preview table 8.) For $3 \mathrm{~g} 1^{`} 2$, two CURR solutions pertain.

\subsection{Elementary particles that do not carry long- range forces}

This unit matches and suggests elementary particles that do not carry long-range forces.

\subsubsection{We develop modeling that matches and sug- gests all elementary particles (other than bosons that intermediate long-range forces) that this essay discusses.}

We explore the notion that solutions for which $\Sigma=0$ associate with known and possible non-Gfamily elementary particles (or, non-LRI elementary particles). Based on arithmetic, for each $\Sigma=0$ solution, $n_{\Gamma}$ is at least three. We associate the symbol SRI (as in short-range interaction or as in elementary boson that does not associate with a long-range interaction) with non-G-family elementary bosons. We associate the symbol ELF (as in elementary fermion) with fermion elementary particles.

Table 5 shows $0 \mathrm{~g} \Gamma$ solutions that might associate with elementary bosons that are not G-family bosons. (Reference [10] discusses the inflaton particle.)

Table 6 shows $0 \mathrm{~g} \Gamma$ solutions that might associate with elementary fermions.

\subsubsection{We discuss modeling that differentiates so- called free aspects of elementary particles from so-called interlaced aspects of elemen- tary particles.}

We discuss a contrast - for non-G-family elementary particles and for more complicated objects between a notion for which this essay uses the word free and a notion for which this essay uses the word interlaced.

Each SRI and ELF elementary particle associates with just one of free and interlaced. Free 
Table 3: PROP and CURR $\Sigma$ ' solutions, for which $l_{\max } \leq 16$ for PROP $\Sigma \mathrm{g}$ ' solutions. Table 3 extends table 2 so as to include - for each PROP $\Sigma g^{\prime}$ solution - a CURR $\Sigma g^{\prime}$ solution. For example $1 \mathrm{~g} 7^{\circ} 8$ satisfies $\Sigma=1$ and associates with - for an object that produces an electromagnetic field - the charge-current 3-vector that complements the scalar charge that associates with PROP and with $1 \mathrm{~g} 1$. The symbol $n_{l_{o}, P R O P}$ denotes the number of elements in the $\Gamma$ that associates with PROP. For a CURR $\Sigma g \Gamma$ solution, the RDP (or, radial dependence of potential) equals $\Xi^{-1}$ times the RDP for the associated PROP $\Sigma \mathrm{g} \Gamma$ solution. Table 3 lists an RDF - or radial dependence of force - for each PROP solution. For each one of PROP and CURR, the RDF equals $\Xi^{-1}$ times the RDP. For example, for each of $1 \mathrm{~g} 1$ and $2 \mathrm{~g} 2$, the RDF is $\Xi^{-2}$, which is $r^{-2}$. Table 3 shows properties - of objects that produce $\Sigma \mathrm{g} \Gamma$ components of $\Sigma \mathrm{g}$ - that associate with the PROP solution. The table attempts to use familiar symbols. The table associates the symbols with phrases. The symbol NYN abbreviates the three-word phrase not yet named and denotes a property for which people might have yet to assign a name. The symbol TBD denotes the three-word phrase to be determined. No one $\Sigma g \Gamma$ solution associates with both PROP and CURR.

\begin{tabular}{|c|c|c|c|c|c|}
\hline$\Sigma$ & $\sum \mathrm{g} \Gamma \mathrm{PROP}$ & $\Sigma g \Gamma \mathrm{CURR}$ & $n_{l_{o}, P R O P}$ & RDF PROP & Properties associating with PROP \\
\hline 1 & 1g1 & $1 \mathrm{~g} 7^{6} 8$ & 1 & $\Xi^{-2}$ & Q - Charge \\
\hline 1 & $\lg 1^{\prime} 2$ & $1 \mathrm{~g} 2^{6} 7^{6} 8$ & 2 & $\Xi^{-3}$ & $\mu$ - Magnetic dipole moment \\
\hline 1 & $\lg 1^{6} 2^{6} 4$ & $1 \lg 1^{6} 2^{6} 4^{6} 8$ & 3 & $\Xi^{-4}$ & $\begin{array}{l}\mu, s \text { - Magnetic dipole moment } \\
\text { and angular momentum }\end{array}$ \\
\hline 1 & $\lg 1^{6} 2^{6} 4^{6} 8^{6} 16$ & $\lg 1^{6} 2^{6} 4^{6} 8^{6} 16^{6} 32$ & 5 & $\Xi^{-6}$ & NYN - TBD \\
\hline 2 & $2 \mathrm{~g} 2$ & $2 g 6^{6} 8$ & 1 & $\Xi^{-2}$ & $m$ - Mass \\
\hline 2 & $2 g 2^{6} 4$ & $2 g 2^{6} 4^{6} 8$ & 2 & $\Xi^{-3}$ & $m, s$ - Rotating mass \\
\hline 2 & $2 \mathrm{~g} 1^{6} 2^{6} 3$ & $2 \mathrm{~g} 1^{6} 2^{6} 3^{6} 8$ & 3 & $\Xi^{-4}$ & $\begin{array}{l}m, \text { NYN - Non-spherical aspects } \\
\text { of mass }\end{array}$ \\
\hline 2 & $\begin{array}{l}2 \mathrm{~g} 1^{6} 2^{6} 3^{6} 4 \mathrm{v} \\
2 \mathrm{~g} 1^{6} 2^{6} 3^{6} 4 \mathrm{w}\end{array}$ & $\begin{array}{l}2 \mathrm{~g} 1^{6} 2^{6} 3^{6} 4^{6} 8 \mathrm{v} \\
2 \mathrm{~g} 1^{6} 2^{6} 3^{6} 4^{6} 8 \mathrm{w}\end{array}$ & 4 & $\Xi^{-5}$ & $\begin{array}{l}m, \text { NYN, } s \text { - Rotating } \\
\text { non-spherical aspects of mass }\end{array}$ \\
\hline 2 & $2 \mathrm{~g} 1^{6} 2^{6} 3^{6} 8^{6} 16$ & $2 \mathrm{~g} 1^{6} 2^{6} 3^{6} 8^{6} 16^{6} 32$ & 5 & $\Xi^{-6}$ & $m$, NYN - TBD \\
\hline 2 & $2 g 1^{`} 2^{`} 3^{`} 4^{`} 8^{6} 16$ & $2 g 1^{6} 2^{6} 3^{6} 4^{6} 8^{6} 16^{6} 32$ & 6 & $\Xi^{-7}$ & $m, \mathrm{NYN}-\mathrm{TBD}$ \\
\hline 3 & $3 g 3$ & $3 \operatorname{gg} 5 \cdot 8$ & 1 & $\Xi^{-2}$ & $\begin{array}{l}\text { NYN - TBD (a function of } \\
\text { elementary fermion flavour) }\end{array}$ \\
\hline 3 & $3 g 2^{6} 3^{6} 4$ & $3 g 2^{6} 3^{6} 4^{6} 8$ & 3 & $\Xi^{-4}$ & NYN - TBD \\
\hline 3 & $3 g 2^{6} 3^{6} 4^{6} 8^{6} 16$ & $3 g 2^{6} 3^{6} 4^{6} 8^{6} 16^{6} 32$ & 5 & $\Xi^{-6}$ & NYN - TBD \\
\hline 4 & $4 \mathrm{~g} 4$ & $4 g 4^{6} 8$ & 1 & $\Xi^{-2}$ & $S$ - Angular momentum \\
\hline 4 & $\begin{array}{l}4 \mathrm{~g} 1^{6} 2^{6} 3^{6} 4 \mathrm{v} \\
4 \mathrm{~g} 1^{6} 2^{6} 3^{6} 4 \mathrm{w}\end{array}$ & $\begin{array}{l}4 \mathrm{~g} 1^{6} 2^{6} 3^{6} 4^{6} 8 \mathrm{v} \\
4 \mathrm{~g} 1^{6} 2^{6} 3^{6} 4^{6} 8 \mathrm{w}\end{array}$ & 4 & $\Xi^{-5}$ & NYN - TBD \\
\hline 4 & $4 \mathrm{~g} 1^{`} 2^{`} 3^{`} 4^{`} 8^{6} 16$ & $4 \mathrm{~g} 1^{6} 2^{6} 3^{6} 4^{6} 8^{6} 16^{6} 32$ & 6 & $\Xi^{-7}$ & NYN - TBD \\
\hline
\end{tabular}

Table 4: Some g-family solutions that associate with $\Sigma$ g"

\begin{tabular}{|c|c|c|c|c|c|}
\hline$\Sigma$ & PROP $\Sigma g \Gamma$ & $\operatorname{PROP} \Sigma=\ldots$ & CURR $\Sigma=\ldots$ & $n_{l_{o}, P R O P}$ & Association \\
\hline 1 & $\lg 3^{6} 4$ & $-3+4$ & $-3-4+8 \mid$ & 2 & Hyperfine interactions \\
\hline 3 & $3 g 1^{6} 2$ & $+1+2$ & $+1-6+8||+2-7+8 \mid$, & 2 & Anomalous magnetic moments \\
\hline
\end{tabular}


Table 5: Solutions that might associate with non-G-family elementary (or, with SRI) bosons. Each column with label $0=\ldots$ shows a calculation that produces the $\Sigma=0$ that associates with a $0 \mathrm{~g} \Gamma$ solution. Each integer that the calculation includes is a member of $\Gamma$. No other integer is a member of $\Gamma$. The symbol $n_{l_{o}, P R O P}$ denotes the number of $l_{o}$ that appear in the $\Gamma$ for the PROP solution. The symbol $n_{E P}$ denotes the number of elementary particles. We posit that the aye (or, 0I boson) associates with notions of an inflaton. Inflatons would be zero-mass zero-charge bosons that might have played key roles during a hypothesized inflationary epoch, early in the evolution of the universe. The $\mathrm{W}$ boson is the only charged elementary boson. In the table, only one PROP solution does not have - as members of $\Gamma$ - all three of 1,3 , and 4 . Based on the previous two sentences, we associate $0 \mathrm{~g} 1^{\prime} 2^{‘}$ ' 3 with the $\mathrm{W}$ boson and we associate $0 \mathrm{~g} 1^{`} 3^{`}{ }^{\prime} 4$ with the $\mathrm{Z}$ boson. We posit that, paralleling aspects of some 1g' CURR solutions, for the CURR solution that associates with the $\mathrm{W}$ boson, $l_{o}=7$ associates with charge. We posit that, paralleling aspects of some 1g' CURR solutions, for the CURR solution that associates with the gluons, $l_{o}=5$ associates with color charge. We posit that the jay (or, $1 \mathrm{~J}$ ) boson associates with notions of Pauli repulsion. Pauli repulsion associates with the notion that two fermions (whether elementary fermions or not elementary fermions) cannot occupy the same state. Pauli repulsion associates with repulsive aspects of the residual strong force. We suggest (but do not require) that - with respect to the Standard Model notion that $S U(3)$ symmetry associates with the strong interaction - the jay boson might (for some modeling) associate with the identity operator that $S U(3)$ representations for the gluons (which transmit color charge) exclude. We suggest that mutual - for gluons and jay bosons - association with the strong force associates with the notion that the $1 \mathrm{~J}$ PROP solution equals the $1 \mathrm{U}$ PROP solution. We suggest that the jay boson can interact with each fermion elementary particle, regardless of whether the notion of color charge pertains for the elementary fermion. We suggest that the jay boson can interact with each fermion non-elementary particle. Each one of two uses of a pair of the symbol ${ }^{\dagger}$ points to dual use of a solution.

\begin{tabular}{llllll}
\hline $0=\ldots$, re $0 \mathrm{~g} \Gamma$ for PROP & $0=\ldots$, re $0 \mathrm{~g} \Gamma$ for CURR & $n_{l_{o}, P R O P}$ & Family & Bosons & $n_{E P}$ \\
\hline$|+1-2-3+4|$ & $|+1-2-3-4+8|$ & 4 & $0 \mathrm{H}$ & Higgs & 1 \\
$|+1-2-3-4-8+16|$ & $|+1-2-3-4-8-16+32|^{\dagger}$ & 6 & $0 \mathrm{I}$ & Aye & 1 \\
$|-1-3+4|$ & $|-1-3-4+8|$ & 3 & $1 \mathrm{Z}$ & $\mathrm{Z}$ & 1 \\
$|-1-2+3|$ & $|+2-3-7+8|$ & 3 & $1 \mathrm{~W}$ & $\mathrm{~W}$ & 1 \\
$|-1-3-4-8+16|^{\dagger}$ & $|-1+2-4-5-8-16+32|$ & 5 & $1 \mathrm{U}$ & Gluons & 8 \\
$|-1-3-4-8+16|^{\dagger}$ & $|+1-2-3-4-8-16+32|^{\dagger}$ & 5 & $1 \mathrm{~J}$ & Jay & 1 \\
\hline
\end{tabular}

Table 6: Solutions that might associate with elementary fermions (or, ELF elementary particles). We posit, regarding PROP $0 \mathrm{~g}$ solutions, that $6 \in \Gamma$ associates with elementary fermions. (Regarding bosons and the CURR 2g6' 8 solution, $6 \in \Gamma$ associates with mass. Otherwise, regarding boson $\Sigma \mathrm{g} \Gamma$ solutions for which $\Sigma \geq 1,6 \notin \Gamma$ pertains. We suggest that each elementary fermion has positive mass.) We posit that, in effect, each PROP solution associates with three flavours and thus with three elementary fermions. Paralleling notions pertaining to non-G-family elementary bosons, if, and only if, each of 1,3 , and 4 is a member of $\Gamma$, an elementary particle that associates with table 6 has zero charge. We discuss solutions that associate with quarks. 0.5Q particles are the only known particles for which $0<Q<1$. Here, $Q$ denotes the magnitude of the charge, in units of $\left|q_{e}\right| . q_{e}$ denotes the charge of the electron. The notion of PROP solution associates with each of the following two solutions: $0=|+1-3-6-8+16|$ and $0=|+2-4-6-8+16|$. The notion of CURR solution associates with each of the following two solutions: $0=|+1-3-6-8-16+32|$ and $0=|+2-4-6-8-16+32|$. Two possibilities exist. For one possibility, the following two sentences pertain. Quarks with one magnitude of charge associate with one of the PROP solutions and one of the CURR solutions. Quarks with the other magnitude of charge associate with the other one of the PROP solutions and the other one of the CURR solutions. For the other possibility, the following three sentences pertain. One of the PROP solutions and one of the CURR solutions associate (for modeling purposes) with $Q=1 / 2$. The other one of the PROP solutions and the other one of the CURR solutions associate (for modeling purposes) with $Q=1 / 6$. One linear combination of the two pairs associates with $Q=(1 / 2)+(1 / 6)=2 / 3$ and another linear combination of the two pairs associates with $Q=(1 / 2)-(1 / 6)=1 / 3$. (Perhaps, preview aspects, such as $Q=1 / 2$, of table 11 and table 12.)

\begin{tabular}{|c|c|c|c|c|c|}
\hline $0=\ldots$, re $0 \mathrm{~g} \Gamma$ for PROP & $0=\ldots$, re $0 \mathrm{~g} \Gamma$ for $\mathrm{CURR}$ & $n_{l_{o}, P R O P}$ & Families & Fermions & $n_{E P}$ \\
\hline$|-1+2-3-4+6|$ & $+1-2+3-4-6+8 \mid$ & 5 & $0.5 \mathrm{~N}$ & Neutrinos & 3 \\
\hline$-1-2-3+6$ & $+2+3-6-7+8$ & 4 & $0.5 \mathrm{C}$ & Charged leptons & 3 \\
\hline$+1-3-4+6 \mid$ & $-1+3-4-6+8$ & 4 & $0.5 \mathrm{~N}^{\prime}$ & Heavy neutrinos & 3 \\
\hline$|+1-3-6-8+16|$ & $|+1-3-6-8-16+32|$, & 5 & $0.5 \mathrm{Q}_{1 / 3}, 0.5 \mathrm{Q}_{2 / 3}$ & Quarks & 6 \\
\hline $\mid \begin{array}{l}|+2-4-6-8+16| \\
-1+3-4-6-8+16 \mid\end{array}$ & $\begin{array}{l}\mid+2-4-6-8-16+32 \\
\mid-1+3-4-6-8-16+32\end{array}$ & 6 & $0.5 \mathrm{R}$ & Arcs & 3 \\
\hline
\end{tabular}


pertains if $l_{\max }=8$ for the CURR solution. Interlaced pertains if $l_{\max }=32$ for the CURR solution.

We suggest that the notion of $l_{\max }=8$ for the CURR solution associates with the possibility that an object models as having a well-defined and constant momentum. We suggest that the notion of $l_{\max }=32$ for the CURR solution associates with the possibility that an object models as existing within a system (of more than one object) that models as having a well-defined and constant momentum. For $l_{\max }=32$ for the CURR solution, the object does not necessarily model as having a well-defined and constant momentum.

\subsubsection{We note some possible modeling that this es- say does not emphasize.}

This essay does not emphasize the combined notion of the extents to which modeling might feature notions of free and interlaced for each of momentum $\left(l_{o}=8\right)$, angular momentum $\left(l_{o}=4\right)$, and $\operatorname{mass}\left(l_{o}=2\right)$.

This essay does not emphasize the notion of extending the following series to address a case that includes $l_{o}=8$ and $l_{o}=16$. For PROP solutions for which $\Sigma=1$ and $1 \in \Gamma, l_{o}=1$ associates with charge. For PROP solutions for which $\Sigma=2$ and $2 \in \Gamma, l_{o}=2$ associates with mass. For PROP solutions for which $1 \leq \Sigma \leq 4$ and $4 \in \Gamma, l_{o}=4$ associates with angular momentum (or with angular velocity). (No PROP solutions satisfy either $8 \in \Gamma$ and $16 \notin \Gamma$ or $8 \notin \Gamma$ and $16 \in \Gamma$.)

\subsection{Isomers and dark matter}

This unit suggests that most dark matter consists of five isomers of most of the ordinary matter elementary particles that are not G-family elementary particles.

\subsubsection{We discuss the notion that, if nature includes only one isomer of each elementary particle, modeling might not suffice to explain known data about dark matter.}

Discussion above points to two types of elementary particles that would measure as dark matter or that would provide a basis for dark matter. $0.5 \mathrm{~N}$ ' fermions associate with the notion of free and would measure as dark matter. 0.5R fermions associate with the notion of interlaced. Hadron-like particles containing gluons and $0.5 \mathrm{R}$ fermions would contain no charged particles and would measure as dark matter.

We use the term DMAI to denote stuff that has bases in $0.5 \mathrm{~N}$ ' particles or $0.5 \mathrm{R}$ elementary fermions. DM abbreviates the two-word term dark matter. AI abbreviates the two-word term all isomers. (Here, we allude to a notion of multiple isomers of some elementary particles. For the moment we assume that nature includes just one isomer.)
We use notation of the form DM:OM to denote an inferred ratio of DM effects to OM effects. OM abbreviates the two-word term ordinary matter.

Table 7 lists some observed ratios of dark matter effects to ordinary matter effects. (For data and discussion regarding densities of the universe, see reference [8]. For data and discussion regarding galaxy clusters, see references [26], [27], [28], and [29]. For data and discussion regarding absorption of $\mathrm{CMB}$, see references [30], 31], and [32]. For data and discussion regarding observed early galaxies, see references [33] and [34]. For data and discussion regarding later galaxies for which ratios of $5^{+}: 1$ pertain, see reference [35]. For data and discussion regarding galaxies for which ratios of $\sim 4: 1$ pertain, see references [36] and 37. For data and discussion regarding the combination of $0^{+}: 1$ and later, see references [38, 39], 40], 41, 42], and [43]. For data and discussion regarding observed dark matter galaxies, see references [35], [44], and [45]. References [46] and [47] suggest, regarding galaxy clusters, the existence of clumps of dark matter that might be individual galaxies. Current techniques might not be capable of observing early dark matter galaxies.)

While there might not be all that much data regarding ratios of other than $5^{+}: 1$ and $1: 0^{+}$, table 7 might point to seemingly prevalent approximate DM:OM ratios of $1: 1,0^{+}: 1$, and $\sim 4: 1$. We suggest that, if DMAI is the only type of dark matter, DMAI might not suffice to explain ratios of dark matter to ordinary matter. We suggest that the notion of DMAI might not suffice to explain dark matter.

\subsubsection{We discuss the notion that nature includes six isomers of each elementary particle that does not intermediate a long-range force.}

We suggest that nature includes six isomers of the SRI and ELF elementary particles - or, six isomers of the set of elementary particles that associates with all non-G-family elementary bosons and all elementary fermions. (See table 5 and table 6.) One isomer associates with ordinary matter plus one isomer of DMAI. That one isomer of DMAI measures as dark matter. Each one of the other five isomers of the set of non-G-family elementary particles measures as dark matter. Regarding densities of the universe, the five isomers of non-DMAI that measure as dark matter associate with the 5 in the DM:OM ratio of $5^{+}: 1$. The six isomers of DMAI associate with the ${ }^{+}$in the DM:OM ratio of $5^{+}: 1$.

We use a two-word phrase isomer number to denote one isomer. Here, number can be any one of zero, one, ..., and five. We associate the two-word term isomer zero with the isomer that includes ordinary matter. We use the two-word phrase alt isomer to denote any one of the five isomers that does not 
Table 7: Ratios of dark matter to ordinary matter. DM denotes dark matter. OM denotes ordinary matter. The notion of DM:OM pertains. Inferences come from interpreting data. The word early associates with the notion that people consider the data to pertain regarding early in the history of the universe. (Redshifts $z$ tend to exceed seven, but could be somewhat less.) The word later associates with the notion that observations pertain to objects later in the history of the universe. In table 7, the four-word phrase some absorption of CMB associates with the notion that people measured some specific depletion of CMB (or, cosmic microwave background radiation) and inferred twice as much depletion as people expected based solely on hyperfine interactions with hydrogen atoms. Possibly, half of the depletion associates with DM effects. The three-word phrase dark matter galaxy denotes a galaxy that contains much less ordinary matter than dark matter.

\begin{tabular}{lllll}
\hline DM & OM & Phenomena & Status & Terminology \\
\hline $5^{+}$ & 1 & Densities of the universe & Observed & - \\
$5^{+}$ & 1 & Some galaxy clusters & Observed & - \\
1 & 1 & Some absorption of CMB & Possibly observed & - \\
$0^{+}$ & 1 & Galaxies (early) & Observed & - \\
$5^{+}$ & 1 & Many (later) galaxies & Observed & - \\
$\sim 4$ & 1 & Some (later) galaxies & Observed & - \\
$0^{+}$ & 1 & Some (later) galaxies & Observed & - \\
1 & $0^{+}$ & Some (later) galaxies & Observed & Dark matter galaxies \\
1 & $0^{+}$ & Some (early) galaxies & Not (yet) observed & (Would be) dark matter galaxies \\
\hline
\end{tabular}

associate with ordinary matter.

All six isomers produce and interact with a common notion of gravity. We suggest that one instance of $2 \mathrm{~g} 2$ mediates interactions between all six isomers. We say that one instance of $2 \mathrm{~g} 2$ has a reach of six, as in six isomers. We suggest that each isomer associates with its own instance of $\lg 1$ and its own instance of $1 \mathrm{~g} 1^{\prime} 2$. We say that each instance of $1 \mathrm{~g} 1$ has a reach of one, as in one isomer. Each instance of $1 \mathrm{~g} 1^{\prime} 2$ has a reach of one. Each isomer - including the ordinary matter isomer - scarcely interacts with any other isomer via electromagnetism.

We address the topic of reach for each $\Sigma \mathrm{g} \Gamma$ to which table 1 alludes. Based on the reach of $1 \mathrm{~g} 1$ and the reach of $1 \mathrm{~g} 1^{6} 2$, we suggest that $n_{o 0}=0$ associates with a reach of one. Based on the reach of $2 \mathrm{~g} 2$, we suggest that $n_{o 0}=1$ associates with a reach of six. We posit that, for $n_{o 0} \geq 1$, the reach (of one instance of a relevant PROP $\Sigma \mathrm{g} \Gamma$ ) equals the number of generators of the group $S U(7)$ divided by the number of generators of the group $S U\left(2 n_{o 0}+1\right)$. For an integer $l$ that is at least two, the number of generators of the group $S U(l)$ is $l^{2}-1$. The reach that associates with $n_{o 0}=2$ is two. The reach that associates with $n_{o 0}=3$ is one. The number of instances of a PROP $\Sigma \mathrm{g} \Gamma$ component of a $\Sigma \mathrm{G}$ G-family elementary particle is six divided by the reach that associates with the PROP $\Sigma \mathrm{g} \Gamma$ solution.

We assume that the reach of a CURR counterpart solution to a PROP $\Sigma g \Gamma$ solution is the same as the reach of the PROP $\Sigma \mathrm{g} \Gamma$ solution.

We address the reach of the $2 \mathrm{~g} 1^{\prime} 2^{`} 3^{\circ} 8^{\prime} 16$ PROP solution, which table 3 lists and table 1 does not list. For $2 \mathrm{~g}^{6} 2^{\text {‘ }} 3^{6} 8^{6} 16$, each of 1 , 2, and 3 appears in $\Gamma$ and 4 does not appear in $\Gamma$. We assume that $n_{o 0}=1$. The reach for $2 \mathrm{~g} 1^{6} 2^{6} 3^{6} 8^{6} 16$ is six.

Table 8 shows the reach $\left(\rho_{I}\right)$ for - and other information about - each one of some solutions that table 2 , table 3 , and table 4 list.
Regarding the notion of a reach, $\rho_{I}$, of two, there are three instances of the PROP solution. We number the isomers so that one instance of the solution intermediates interactions between isomer zero and isomer three. One instance of the solution intermediates interactions between isomer one and isomer four. One instance of the solution intermediates interactions between isomer two and isomer five.

We use notation of the form $\Sigma\left(\rho_{I}\right) \mathrm{g} \Gamma$ to denote a $\Sigma \mathrm{g} \Gamma$ solution and the reach $\rho_{I}$ that associates with one modeling use that features an instance of the solution. For example, 2(2)g2 44 pertains regarding $2 \mathrm{~g} 2^{\circ} 4$. We extend use of such notation to non-LRI elementary particles. For non-LRI elementary particles, the reach is one and notation of the form $S(1) \Phi$ pertains.

\subsubsection{We start to discuss the extent to which the properties of any one isomer's elementary particles differ from the properties of elemen- tary particles that associate with other iso- mers.}

If the stuff that associates with each of the five all-dark-matter isomers evolved similarly to ordinary matter, our suggestions regarding dark matter might not adequately comport with observations regarding the Bullet Cluster collision of two galaxy clusters. Elsewhere, we suggest that the isomers of ELF elementary particles differ sufficiently that our suggestions regarding dark matter do not necessarily disagree with observations pertaining to the Bullet Cluster. (Perhaps, preview discussion related to table 14.)

\subsubsection{We discuss notions regarding excitation and de-excitation of LRI fields (or, of G-family elementary particles).}

An excitation associates with a value of $\Sigma$ and with a set of isomers. For example, consider an 
Table 8: Reaches and other information regarding some solutions that associate with electromagnetism, gravity, 3G, and 4G. $\rho_{I}$ denotes reach. $\Sigma \in \Gamma$ associates with the symbol g'. $\Sigma \notin \Gamma$ associates with the symbol g". NYN denotes not yet named. Discussion related to table 19 provides information regarding the notion of anomalous magnetic moment.

\begin{tabular}{|c|c|c|c|c|c|c|}
\hline$S$ & $\Sigma$ & PROP solution & $\rho_{I}$ & Solution type & PROP RDF & Object property \\
\hline 1 & 1 & 1g1 & 1 & $\Sigma \in \Gamma$ & $\Xi^{-2}$ & Charge \\
\hline 1 & 1 & $\lg 1^{6} 2$ & 1 & $\Sigma \in \Gamma$ & $\Xi^{-3}$ & Magnetic dipole moment \\
\hline 1 & 1 & $\lg 1^{6} 2^{6} 4$ & 6 & $\Sigma \in \Gamma$ & $\Xi^{-4}$ & NYN \\
\hline 1 & 1 & $\lg 3^{6} 4$ & 2 & $\Sigma \notin \Gamma$ & $\Xi^{-3}$ & NYN \\
\hline 2 & 2 & $2 g 2$ & 6 & $\Sigma \in \Gamma$ & $\Xi^{-2}$ & Mass \\
\hline 2 & 2 & $2 \mathrm{~g} 2^{6} 4$ & 2 & $\Sigma \in \Gamma$ & $\Xi^{-3}$ & NYN \\
\hline 2 & 2 & $2 \mathrm{~g} 1^{6} 2^{6} 3$ & 1 & $\Sigma \in \Gamma$ & $\Xi^{-4}$ & NYN \\
\hline 2 & 2 & $2 \mathrm{~g} 1^{6} 2^{6} 3^{6} 4 \mathrm{v}$ & 1 & $\Sigma \in \Gamma$ & $\Xi^{-5}$ & NYN \\
\hline 2 & 2 & $2 \mathrm{~g} 1^{6} 2^{6} 3^{6} 4 \mathrm{w}$ & 1 & $\Sigma \in \Gamma$ & $\Xi^{-5}$ & NYN \\
\hline 2 & 2 & $2 \mathrm{~g} 1^{6} 2^{6} 3^{6} 8^{6} 16$ & 6 & $\Sigma \in \Gamma$ & $\Xi^{-6}$ & NYN \\
\hline 3 & 3 & $3 g 3$ & 2 & $\Sigma \in \Gamma$ & $\Xi^{-2}$ & NYN \\
\hline 3 & 3 & $3 g 2^{6} 3^{6} 4$ & 6 & $\Sigma \in \Gamma$ & $\Xi^{-4}$ & NYN \\
\hline 3 & 3 & $3 g 1^{6} 2$ & 1 & $\Sigma \notin \Gamma$ & $\Xi^{-3}$ & Anomalous magnetic moment \\
\hline 4 & 4 & $4 \mathrm{~g} 4$ & 1 & $\Sigma \in \Gamma$ & $\Xi^{-2}$ & Angular momentum \\
\hline 4 & 4 & $4 \mathrm{~g} 1^{6} 2^{‘} 3^{6} 4 \mathrm{v}$ & 1 & $\Sigma \in \Gamma$ & $\Xi^{-5}$ & NYN \\
\hline 4 & 4 & $4 \mathrm{~g} 1^{6} 2^{6} 3^{6} 4 \mathrm{w}$ & 1 & $\Sigma \in \Gamma$ & $\Xi^{-5}$ & NYN \\
\hline
\end{tabular}

excitation that associates with active-gravitational properties of an ordinary matter star. The word active associates with the notion that the star generates gravity. The word gravitational associates with $\Sigma=2$. The excitation might associate with the $2 \mathrm{~g} 2$ solution, with the $2 \mathrm{~g}^{4} 4$ solution, or with another $2 \mathrm{~g}$ solution. Because the star consists just of ordinary matter stuff, the set of isomers consists just of isomer zero.

A de-excitation associates with the notion of passive properties, with any same- $\Sigma$ G-family solution and with a set of isomers that associates with the original excitation. We continue the previous example. Regarding $\Sigma=2$, the word passive associates with the notion that an object interacts with gravity that other objects actively produce. Because $2 \mathrm{~g} 2$ has a reach of six, any object can deexcite, via $2 \mathrm{~g} 2$, the excitation that the example features. Because $2 \mathrm{~g} 1$ ' 2 ' 3 has a reach of one, only isomer zero stuff can de-excite, via $2 \mathrm{~g} 1^{`} 2^{`} 3$, the excitation. Because $2 \mathrm{~g} 2^{6} 4$ has a reach of two, only isomer zero stuff or isomer three stuff can de-excite the excitation via $2 \mathrm{~g}^{\circ} 44$.

Generally, ten types of de-excitations exist. One type consists of de-excitations that associate with reach-six solutions. Three types consist of deexcitations that associate with reach-two solutions. One of the three types associates with isomer zero and isomer three. Another one of the three types associates with isomer one and isomer four. The other one of the three types associates with isomer two and isomer five. Six types consist of de-excitations that associate with reach-one solutions. Each one of the six types associates with exactly one isomer.

We discuss excitations and de-excitations that associate with long-range forces produced by a galaxy that consists mainly of stuff that associates with five isomers. (Perhaps, see table 7.) We discuss electromagnetism. A one-isomer distant observer would sense mostly aspects that associate with the distant observer's isomer. Here, most detection of photons would associate with the reachone solutions $1 \mathrm{~g} 1$ and $1 \mathrm{~g} 1^{\prime} 2$. Via aspects that associate with solutions such as $1 \mathrm{~g} 1^{\circ} 2^{\circ} 4$ (for which the reach is six) and $1 \mathrm{~g} 33^{4} 4$ (for which the reach is two), the one-isomer observer might sense aspects that associate with isomers other than the observer's isomer. We discuss gravitation. The one-isomer distant observer would sense all of the galaxy's stuff via $2 \mathrm{~g} 2$ (for which the reach is six). But the sensing of subtleties (such as rotation of stuff or irregular distributions of stuff) associates with solutions (such as $2 \mathrm{~g} 2^{\prime} 4$ and $2 \mathrm{~g} 1^{\prime} 2^{\prime} 3$ ) for which the reaches are less than six. (Perhaps, see table 8.) The oneisomer observer would not necessarily sense directly via $2 \mathrm{G}$ all of the subtleties.

\section{Results}

This unit discusses explanations of known data and discusses suggestions regarding possible data that people have not yet measured. The discussion includes explanations and suggestions regarding elementary particles, dark matter, galaxies, and the cosmos.

\subsection{Explanations and suggestions regarding ele- mentary particles}

This unit lists elementary particles that associate with our modeling and discusses relationships between properties of elementary particles. 


\subsubsection{We list all elementary particles of which peo- ple know or that we suggest.}

Table 9 consolidates and summarizes information about all elementary particles of which people know or that this essay suggests. (See table 3, table 5 , and table 6.)

\subsubsection{We explore relationships among properties of objects, elementary particles, and long-range interactions.}

Table 10 discusses relationships between properties of elementary bosons. (Regarding the masses of the Higgs, Z, and W bosons, we used data that reference [8] provides.)

Table 10 points to possibly deeper (than people might otherwise suggest) relationships between the physics properties of spin, mass, and charge.

We turn our attention to properties of elementary fermions.

We consider hypothetical elementary fermions for which $Q=1$. For some value of mass, the gravitational attraction between two identical such hypothetical elementary fermions would equal the electrostatic repulsion between the two fermions. Our work shows that a mass - so-called $m(18,3)$ - seems to have meaning beyond the notion that - for the mass $m(18,3)$ - gravitational attraction between two $Q=1$ identical elementary fermions would be three-quarters of the electrostatic repulsion between the two identical elementary fermions. (Perhaps, preview table 13.)

Table 11 discusses relationships between properties of known charged elementary fermions. (Reference [8] provides the data that underlies table 11.)

Table 12 shows equations that underlie aspects of table 11. (Reference [8] provides the data that underlies table 12.)

\subsubsection{We discuss the masses of neutrinos and of elementary fermions that we suggest.}

Table 13 suggests extensions - for example, regarding $0.5 \mathrm{~N}$ neutrinos and $0.5 \mathrm{R}$ arcs - to table 11 . (Reference [8] provides the data that underlies table 13.)

Table 11 and table 12 point to possibly deeper (than people might otherwise suggest) relationships between the physics properties of spin, mass, and charge.

We explore two alternatives regarding values of $d^{\prime}(0), d^{\prime}(1)$, and $d^{\prime}(2)$. (See table 12.) Changing those numbers would impact only the calculated masses for quarks and the calculated suggested masses for arcs. In both cases, if one excludes one of three methods for estimating the mass of the top quark, the calculated mass for each of the six quarks is within five standard deviations of the experimental mass. (Reference [8] discusses the three methods.) For the third method for estimating the mass of the top quark, the value that we calculate for the mass of the top quark would be less than eleven standard deviations below the mass people have calculated.

One alternative has bases in the notions of $d^{\prime}(-1)=0^{2} / 2^{2}, d^{\prime}(0)=1^{2} / 2^{2}, d^{\prime}(1)=-2^{2} / 2^{2}$, and $d^{\prime}(2)=-(2 \times 3) / 2^{2}$. For this alternative, the three arc rest energies would, respectively, be $\approx 8.14 \mathrm{MeV}, m(1,3) c^{2}$, and $m(2,3) c^{2}$.

The other alternative has bases in the notions of $d^{\prime}(0) \approx 0.264825, d^{\prime}(1)=-2^{2} / 2^{2}$, and $d^{\prime}(2)=$ $-(2 \times 3) / 2^{2}$. For this alternative, the three arc rest energies would, respectively, equal $m(1,3) c^{2}$, $m(1,3) c^{2}$, and $m(2,3) c^{2}$. Across the three $0.5 \mathrm{C}$ elementary fermions and the three $0.5 \mathrm{R}$ elementary fermions, $m(0,3) c^{2}$ would pertain once, $m(1,3) c^{2}$ would pertain twice, $m(2,3) c^{2}$ would pertain twice, and $m(3,3) c^{2}$ would pertain once.

We speculate regarding possible masses for heavy neutrinos.

For purposes of estimating or calculating masses, neutrinos associate with a value of $M^{\prime \prime}$ for which $-6 \leq M^{\prime \prime} \leq-3$. Charged leptons associate with $0 \leq M^{\prime \prime} \leq 3$. If heavy neutrinos associate with $6 \leq M^{\prime \prime} \leq 9$, a lower bound on rest energies for heavy neutrinos might be $m(6,3) c^{2} \sim 6 \times 10^{3} \mathrm{GeV}$, which might be large enough to comport with limits that associate with observations. (References [48 and 49] discuss limits that observations may set. People have not detected $0.5 N^{\prime}$ particles.)

\subsection{Explanations and suggestions regarding dark matter}

This unit suggests specifications for dark matter.

\subsubsection{We discuss - for the six isomers - elementary-fermion masses, flavours, and handedness.}

Regarding each $l$ that is at least one, we assume that the elementary particles in isomer $l$ match with respect to mass - the elementary particles in isomer zero.

For $0 \leq l \leq 5$, we associate the quarks in isomer $l$ with three values of $M^{\prime \prime}$. (See table 11 and table 12.) The values are $3 l+0,3 l+1$, and $3 l+2$. Across the six isomers, quarks associate with each value of $M^{\prime \prime}$ that is in the range $0 \leq M^{\prime \prime} \leq 17$. Regarding quarks and flavours, we assume that - within isomer $l$ - flavour 1 associates with $M^{\prime \prime}=3 l$, flavour 2 associates with $M^{\prime \prime}=3 l+1$, and flavour 3 associates with $M^{\prime \prime}=3 l+2$.

Aspects of table 11 and table 12 point to the possibility that means for matching flavours and masses for charged leptons do not match means for matching flavours and masses for quarks. For charged leptons, isomer zero does not have a charged lepton that associates with $M^{\prime \prime}=1$ and does have a charged lepton that associates with $M^{\prime \prime}=3$. We 
Table 9: Elementary particles. The symbol $Q$ associates with magnitude of charge. The columns labeled $Q>0$ and $Q=0$ have entries in the form of a name of one particle or a name of a set of more than one particle, followed by (in parentheses) a number of particles, followed by a family. NYN denotes not yet named. NYD denotes not yet detected. One might assert that people know of some NYD particles, at least indirectly. For purposes of this essay, PROP $8 \notin \Gamma$ associates with a notion of can model as not interlaced (or, as free) and PROP $8 \in \Gamma$ associates with a notion of always models as interlaced (or, bound).

\begin{tabular}{lllllll}
\hline$S$ & $m$ & $Q>0$ & $Q=0$ & Status & $\Sigma$ & PROP $8 \in \Gamma$ \\
\hline 0 & $>0$ & - & Higgs boson $(1)-0 \mathrm{H}$ & Known & 0 & No \\
$1 / 2$ & $>0$ & Charged leptons $(3)-0.5 \mathrm{C}$ & Neutrinos $(3)-0.5 \mathrm{~N}$ & Known & 0 & No \\
$1 / 2$ & $>0$ & - & Heavy neutrinos $(3)-0.5 \mathrm{~N}$ & NYD & 0 & No \\
1 & $>0$ & W boson $(1)-1 \mathrm{~W}$ & Z boson $(1)-1 \mathrm{Z}$ & Known & 0 & No \\
1 & $=0$ & - & Photon $(1)-1 \mathrm{G}$ & Known & 1 & Some components \\
2 & $=0$ & - & Graviton $(1)-2 \mathrm{G}$ & NYD & 2 & Some components \\
3 & $=0$ & - & NYN $(1)-3 \mathrm{G}$ & NYD & 3 & Some components \\
4 & $=0$ & - & NYN $(1)-4 \mathrm{G}$ & NYD & 4 & Some components \\
0 & $=0$ & - & Aye boson $(1)-0 \mathrm{I}$ & NYD & 0 & Yes \\
$1 / 2$ & $>0$ & Quarks $(3)-0.5 \mathrm{Q}_{1 / 3}$ & - & Known & 0 & Yes \\
$1 / 2$ & $>0$ & Quarks $(3)-0.5 \mathrm{Q}_{2 / 3}$ & - & Known & 0 & Yes \\
$1 / 2$ & $>0$ & - & Arcs $(3)-0.5 \mathrm{R}$ & NYD & 0 & Yes \\
1 & $=0$ & - & Jay boson $(1)-1 \mathrm{~J}$ & NYD & 0 & Yes \\
1 & $=0$ & - & Gluons $(8)-1 \mathrm{U}$ & Known & 0 & Yes \\
\hline
\end{tabular}

Table 10: Relationships between properties of elementary bosons. $Q$ denotes the magnitude of charge, in units of $\left|q_{e}\right|$. $m$ denotes mass, in units of $m_{\text {Higgs }} / 17^{1 / 2}$ or in units of $m_{\mathrm{Z}} / 9^{1 / 2}$. $S$ denotes spin, as in the expression $S(S+1) \hbar^{2}$. $l_{m}$ equals -1 for $m>0$ and equals 0 for $m=0$. The sum is the sum of the numbers in the preceding four columns. Each sum is the square of an integer. For each nonzero mass particle, the integer equals $n_{l_{o}, P R O P}$. There are no nonzero mass elementary bosons for which the integer equals one or two. (For a $\Gamma$ that includes just one value of $l_{o}$ or that includes just two values of $l_{o}, \Sigma \neq 0$ pertains.) NYN denotes the three-word phrase not yet named. Of the non-zero masses to which table 10 alludes, the most accurately known mass is that of the $\mathrm{Z}$ boson. Using the mass of the $\mathrm{Z}$ boson and numbers in table 10 , one can calculate a nominal mass for the Higgs boson and a nominal mass for the W boson. The calculated mass for the Higgs boson differs from the experimentally determined mass by less than two (experimental) standard deviations. The calculated mass for the $\mathrm{W}$ boson differs from the experimentally determined mass by less than four (experimental) standard deviations. To the extent that one uses the notion that ruling out an equality requires a difference of at least five standard deviations, experimental results do not seem to rule out relationships that table 10 states. NYN denotes not yet named.

\begin{tabular}{lllllll}
\hline Bosons & Family & $Q(Q+1)$ & $m^{2}$ & $S^{2}$ & $l_{m}$ & Sum \\
\hline Higgs & 0H & 0 & 17 & 0 & -1 & 16 \\
Aye & OI & 0 & 0 & 0 & 0 & 0 \\
Z & $1 \mathrm{Z}$ & 0 & 9 & 1 & -1 & 9 \\
W & $1 \mathrm{~W}$ & 2 & 7 & 1 & -1 & 9 \\
Jay & $1 \mathrm{~J}$ & 0 & 0 & 1 & 0 & 1 \\
Gluons & $1 \mathrm{U}$ & 0 & 0 & 1 & 0 & 1 \\
Photon & $1 \mathrm{G}$ & 0 & 0 & 1 & 0 & 1 \\
Graviton & $2 \mathrm{G}$ & 0 & 0 & 4 & 0 & 4 \\
NYN & $3 \mathrm{G}$ & 0 & 0 & 9 & 0 & 9 \\
NYN & $4 \mathrm{G}$ & 0 & 0 & 16 & 0 & 16 \\
\hline
\end{tabular}

Table 11: $\log { }_{10}\left(m_{\text {particle }} / m_{e}\right)$ for known charged elementary fermions. Regarding "flavour," this table generalizes, based on terminology that associates with charged leptons and neutrinos. For example, people use the term electron-neutrino. The "Flavour (0.5C)" terms pertain for fermions in the 0.5C family. The "Flavour (0.5Q)" terms pertain for quarks (or, elementary particles in the two families $0.5 \mathrm{Q}_{2 / 3}$ and $\left.0.5 \mathrm{Q}_{1 / 3}\right)$. $M^{\prime \prime}$ is an integer parameter. The domain $-6 \leq M^{\prime \prime} \leq 18$ might have relevance regarding modeling. $Q$ denotes the magnitude of charge, in units of $\left|q_{e}\right|$. Regarding the rightmost four columns, items show (conceptually) $\log 10\left(m_{\text {particle }} / m_{e}\right)$ and the name of an elementary fermion. For each Calc-D case, no particle pertains. For the Calc-F case, no particle pertains. Regarding $Q=1 / 2(\dagger)$, no elementary particles associate with this column. Each number in this column equals the average of the numbers in the $Q=2 / 3$ column and the $Q=1 / 3$ column. The notion of geometric mean pertains regarding the mass of the $Q=2 / 3$ particle and the mass of the $Q=1 / 3$ particle. Regarding Calc-F, a formula for $m\left(M^{\prime \prime}, M^{\prime}\right)$ calculates this number. Table 12 shows the formula.

\begin{tabular}{lllllll}
\hline Flavour $(0.5 \mathrm{C})$ & Flavour $(0.5 \mathrm{Q})$ & $M^{\prime \prime}$ & $Q=1(0.5 \mathrm{C})$ & $Q=2 / 3\left(0.5 \mathrm{Q}_{2 / 3}\right)$ & $Q=1 / 2(\dagger)$ & $Q=1 / 3\left(0.5 \mathrm{Q}_{1 / 3}\right)$ \\
\hline 1 (Electron) & 1 (Electron) & 0 & 0.00 Electron & $0.66 \mathrm{Up}$ & $0.80($ Calc-D) & 0.94 Down \\
- & $2(\mathrm{Mu})$ & 1 & 1.23 (Calc-F) & 3.36 Charm & 2.83 (Calc-D) & 2.29 Strange \\
$2(\mathrm{Mu})$ & $3(\mathrm{Tau})$ & 2 & 2.32 Muon & 5.52 Top & $4.72($ Calc-D) & 3.92 Bottom \\
$3(\mathrm{Tau})$ & - & 3 & $3.54 \mathrm{Tau}$ & - & - & - \\
\hline
\end{tabular}


Table 12: Equations that underlie aspects of table 11. This table shows equations that may pertain regarding all known charged elementary fermions, the known $0.5 \mathrm{~N}$ neutrinos, and the suggested $0.5 \mathrm{R}$ arcs.

\begin{tabular}{|c|c|}
\hline Topic & Note \\
\hline Preliminary calculation & $\begin{array}{l}\beta^{\prime}=m_{\tau} / m_{e} \text { - Defines } \beta^{\prime} . m_{\tau} \text { equals the mass of the tau particle (which is a } \\
\text { charged lepton). } m_{e} \text { equals the mass of the electron. } \\
(4 / 3) \times\left(\beta^{2}\right)^{6}=\left(\left(q_{e}\right)^{2} /\left(4 \pi \varepsilon_{0}\right)\right) /\left(G_{N}\left(m_{e}\right)^{2}\right) \text { - Defines } \beta \text {. The right-hand side of } \\
\text { the equation is the ratio of the electrostatic repulsion between two electrons to } \\
\text { the gravitational attraction between the two electrons. The ratio does not } \\
\text { depend on the distance between the two electrons. } \\
\beta \approx 3477.1891 \pm 0.0226 \text { - This number results from data and the formula that } \\
\text { defines } \beta \text {. The standard deviation reflects the standard deviation for } G_{N} \text {, the } \\
\text { gravitational constant. } \\
\beta^{\prime}=\beta \text { - We posit this equation. } \\
m_{\tau} \text {, calculated } \approx 1776.8400 \pm 0.0115 \mathrm{MeV} / c^{2}-\text { This number results from data } \\
\text { and from } \beta^{\prime}=\beta \text {. }\end{array}$ \\
\hline ion & $\begin{array}{l}\text { These calculations produce numbers that table } 11 \text { shows. } \\
M^{\prime}=3 Q . \\
m\left(M^{\prime \prime}, M^{\prime}\right)=m_{e} \times\left(\beta^{1 / 3}\right)^{\left.M^{\prime \prime}+\left(j_{M^{\prime \prime}}^{\prime \prime}\right) d^{\prime \prime} \times\left(\alpha^{-1 / 4}\right) g\left(M^{\prime}\right) \cdot\left(1+M^{\prime \prime}\right)+j_{M^{\prime}}^{\prime} d^{\prime}\left(M^{\prime \prime}\right)\right)} . \\
\alpha=\left(\left(q_{e}\right)^{2} /\left(4 \pi \varepsilon_{0}\right)\right) /(\hbar c) \text { - Expression for } \alpha, \text { the fine-structure constant. } \\
j_{M^{\prime \prime}}^{\prime \prime}=0,+1,0,-1 \text { for, respectively, } M^{\prime \prime} \bmod 3=0,1,3 / 2,2 ; \text { with } 3 / 2 \bmod 3 \equiv \\
3 / 2 . \\
d^{\prime \prime}=\left(2-\left(\log \left(m_{\mu} / m_{e}\right) / \log \left(\beta^{1 / 3}\right)\right)\right) \approx 3.840679 \times 10^{-2} . \\
g\left(M^{\prime}\right)=0,3 / 2,3 / 2,3 / 2,3 / 2, \text { for, respectively, } M^{\prime}=3,2,3 / 2,1,0 . \\
j_{M^{\prime}}^{\prime}=0,-1,0,+1,+3 \text { for, respectively, } M^{\prime}=3,2,3 / 2,1,0 . \\
d^{\prime}(0) \sim 0.324, d^{\prime}(1) \sim-1.062, d^{\prime}(2) \sim-1.509-\text { Based on attempting to fit } \\
\text { data. }\end{array}$ \\
\hline
\end{tabular}

assume that - for each $l$ - a charged lepton associates with each of $M^{\prime \prime}=3 l+0, M^{\prime \prime}=3 l+2$, and $M^{\prime \prime}=3 l+3$.

We assume that - for each isomer $l$ such that $1 \leq l \leq 5$ - the charged-lepton flavour that associates with $M^{\prime \prime}=3(l)+0$ equals the flavour that associates with the isomer $l-1$ charged lepton that associates with the same value of $M^{\prime \prime}$ and - thus with $M^{\prime \prime}=3(l-1)+3$. We assume that across the six isomers, one cyclical order pertains regarding flavours for charged leptons.

Table 14 shows, for isomers of charged elementary fermions, matches between masses and flavours.

Beyond the topic of flavours, the topic of handedness exists. Ordinary matter associates with lefthandedness. We suggest the possibility (but we do not necessarily require) that isomers 0,2 , and 4 associate with left-handedness and that isomers 1,3 , and 5 associate with right-handedness.

\subsubsection{We prepare to discuss the evolution of stuff that associates with each isomer.}

We associate the symbol OMSE with all SRI elementary particles and all ELF elementary particles except $0.5 \mathrm{~N}$ ' and $0.5 \mathrm{R}$ elementary particles. OMSE abbreviates the three-element phrase ordinary-matter-similar elementary particles. We associate the symbol DMAI with the $0.5 \mathrm{~N}$ ' and $0.5 \mathrm{R}$ elementary particles. DMAI abbreviates the five-word phrase dark matter regarding all isomers. DMAI associates with the notion that - regarding isomer zero - these particles measure as being dark matter and do not measure as being ordinary matter.

We use the three-element term isomer number stuff to denote objects (including SRI elementary particles, ELF elementary particles, hadron-like particles, clumps of stuff, and stars) that associate with the isomer number set of other-than-SG elementary particles.

\subsubsection{We discuss - for each isomer - the evolution of stuff that associates with that isomer.}

$0.5 \mathrm{R}$ particles model as interlaced. (See table 6.) We suggest that - at least after the inflationary epoch - 0.5R-based stuff consists of hadron-like particles. Each 0.5R-based-stuff hadron-like particle includes gluons and at least two arcs. (We deemphasize discussing roles that jay bosons might play.) Our work does not suggest an extent to which 0.5R-based stuff might form primordial black holes.

$0.5 N^{\prime}$ particles model as free. (See table 6.)

Regarding each one of the six isomers, we suggest that stuff made from DMAI behaves within 
Table 13: Possible extensions to table 11. This table shows equations that may pertain regarding the known $0.5 \mathrm{~N}$ neutrinos and the suggested $0.5 \mathrm{R}$ arcs. This table does not discuss the suggested $0.5 \mathrm{~N}$ ' heavy neutrinos.

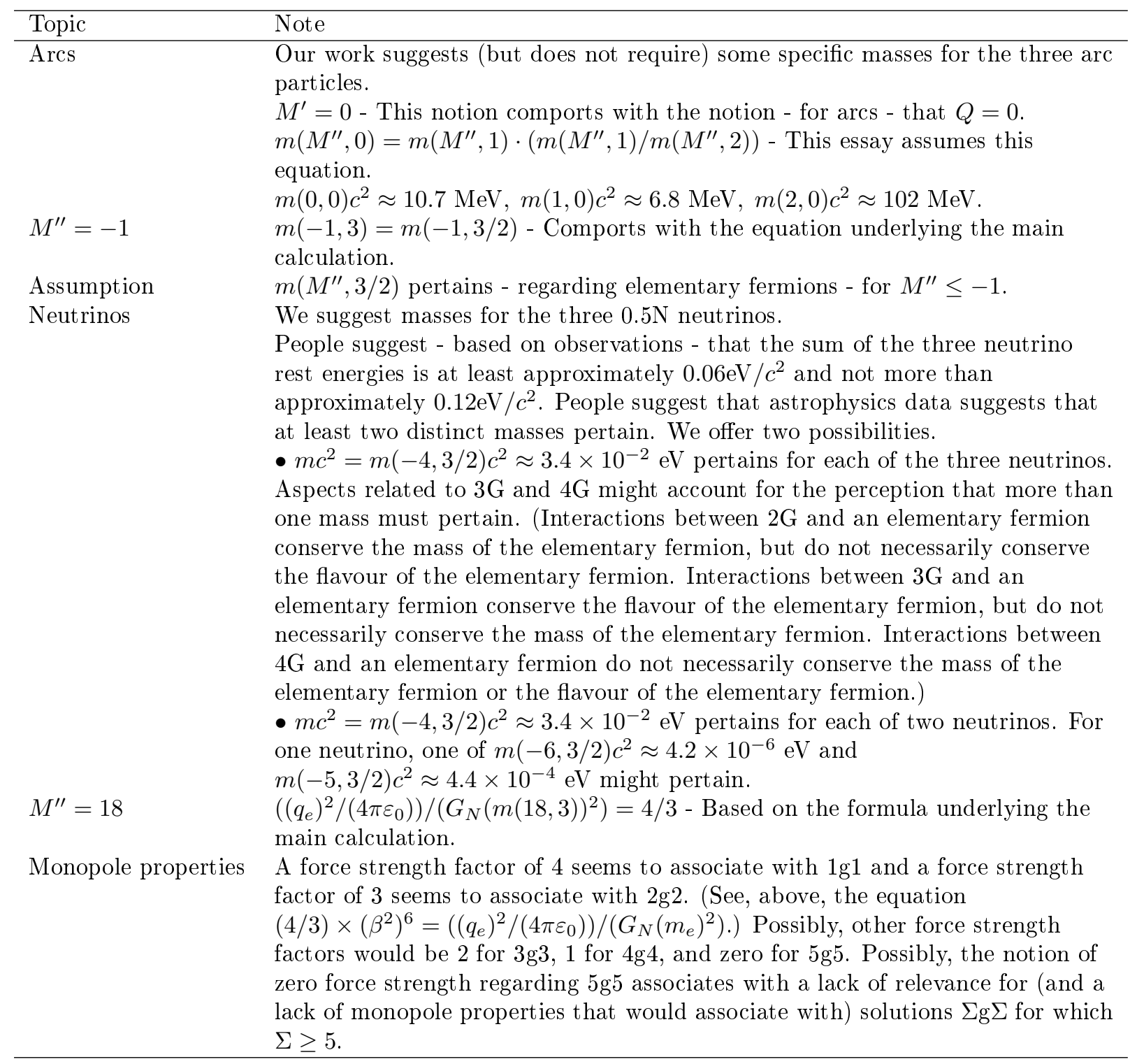

Table 14: Matches between masses and flavours, for isomers of charged elementary fermions. The symbol 0.5Q denotes the pair $0.5 \mathrm{Q}_{1 / 3}$ and $0.5 \mathrm{Q}_{2 / 3}$.

\begin{tabular}{lllll}
\hline Isomer & $M^{\prime \prime}(0.5 \mathrm{Q})$ & Respective flavours $(0.5 \mathrm{Q})$ & $M^{\prime \prime}(0.5 \mathrm{C})$ & Respective flavours $(0.5 \mathrm{C})$ \\
\hline 0 & $0,1,2$ & $1,2,3$ & $0,2,3$ & $1,2,3$ \\
1 & $3,4,5$ & $1,2,3$ & $3,5,6$ & $3,1,2$ \\
2 & $6,7,8$ & $1,2,3$ & $6,8,9$ & $2,3,1$ \\
3 & $9,10,11$ & $1,2,3$ & $9,11,12$ & $1,2,3$ \\
4 & $12,13,14$ & $1,2,3$ & $12,14,15$ & $3,1,2$ \\
5 & $15,16,17$ & $1,2,3$ & $15,17,18$ & $2,3,1$ \\
\hline
\end{tabular}


bounds for dark matter that associate with concordance cosmology.

We discuss the evolution of isomer $1,2,4$, and 5 OMSE stuff.

Here, we use the two-word term alt isomer to designate an isomer other than isomer zero and isomer three.

A charged baryon that includes exactly three flavour 3 quarks is more massive than the counterpart zero-charge baryon that includes exactly three flavour 3 quarks. (For example, two tops and a bottom have a larger total mass than do one top and two bottoms.) Alt isomer flavour 3 charged leptons are less massive than isomer zero flavour 3 charged leptons. When flavour 3 quark states are much populated (and based on interactions mediated by $\mathrm{W}$ bosons), the alt isomer converts more charged baryons to zero-charge baryons than does isomer zero. Eventually, in the alt isomer, interactions that entangle multiple $\mathrm{W}$ bosons result in the alt isomer having more neutrons and fewer protons than does isomer zero. The sum of the mass of a proton and the mass of an alt isomer flavour 1 charged lepton exceeds the mass of a neutron. Compared to isomer zero neutrons, alt isomer neutrons scarcely decay. The IGM (or, intergalactic medium) that associates with the alt isomer scarcely interacts with itself via electromagnetism.

We discuss the evolution of isomer three OMSE stuff.

The following possibilities pertain. The evolution of isomer three OMSE stuff parallels the evolution of ordinary matter (or, isomer zero OMSE stuff). The evolution of isomer three OMSE stuff does not parallel the evolution of ordinary matter (or, isomer zero OMSE stuff). The second possibility might associate with - for example - a difference in handedness - with respect to charged leptons or with respect to $\mathrm{W}$ bosons - between isomer three and isomer zero. (Perhaps note that - regarding SRI and ELF elementary particles - 7 is a member of the CURR $\Gamma$ for, and only for, the $\mathrm{W}$ boson and charged leptons. See table 5 and table 6. .)

\subsection{Explanations and suggestions regarding the rate of expansion of the universe}

This unit suggests eras in the rate of expansion of the universe and suggests mechanisms that associate with the eras.

\subsubsection{We discuss perspective regarding the rate of expansion of the universe.}

Concordance cosmology points to three eras in the so-called rate of expansion of the universe. The eras feature, respectively, rapid expansion; continued expansion, with the rate of expansion decreasing; and continued expansion, with the rate of expansion increasing.
This essay suggests using the notion of eras regarding the separating from each other of clumps - that, today, people would consider to be large of stuff. Examples of such clumps might include galaxy clusters and possibly even larger clumps.

\subsubsection{We provide perspective regarding long-range interactions between objects.}

As two objects move away from each other, the relative effect of an RDF $\Xi^{-(k+1)}$ component decreases compared to the effect of an RDF $\Xi^{-k}$ component. One might associate the two-word phrase time period with a time range in which an $\operatorname{RDF} \Xi^{-l}$ component provides dominant effects. Assuming that objects move away from each other and that one time period associates with $\Xi^{-(k+1)}$ and another time period associates with $\Xi^{-k}$, the time period that associates with $\Xi^{-(k+1)}$ comes before the time period that associates with $\Xi^{-k}$. Two smaller objects (such as galaxies) transit similar time periods more quickly than do two larger objects (such as galaxy clusters).

We consider two objects that are some distance apart. We consider doubling linear dimensions that is doubling the distance between the objects and doubling the diameters of the objects - while maintaining, for each object, a constant mass per unit volume. A PROP RDF $\Xi^{-6}$ force after the doubling of linear dimensions equals the PROP RDF $\Xi^{-6}$ force before the doubling of linear dimensions. Possibly, this invariance regarding scaling suggests reasons not to pursue - based on aspects that this essay suggests - modeling regarding PROP $\mathrm{RDF} \Xi^{-l}$ for which $l$ exceeds six.

\subsubsection{We discuss known and suggested eras in the history of the universe.}

Table 15 discusses eras in the rate of separating of large clumps. (For discussion about the possible inflationary epoch, see references [50] and [10]. For data and discussion about the two multi-billionyears eras, see references [51, [52, [53, and [54. For data and discussion about the possibility that concordance cosmology modeling underestimates for the second multi-billion-years era - increases in the rate of separation, see references [18], [19], [20], [21], [55, [56, and [57.)

Table 16 suggests details regarding eras to which table 15 alludes.

Before inflation, boson PROP solutions for which $\Sigma \geq 2$ and $8 \in \Gamma$ associate with dominant long-range effects. The word interlaced associates with those PROP solutions. After inflation, compared to boson PROP solutions for which $\Sigma \geq 2$ and $8 \notin \Gamma$, boson PROP solutions for which $\Sigma \geq 2$ and $8 \in \Gamma$ do not associate with significant long-range effects. Boson PROP solutions for which $\Sigma=0$ and $8 \in \Gamma$ continue to associate with relevant effects, 
Table 15: Eras regarding the rate of separating of large clumps. The rightmost three columns suggest eras. Subsequent rows associate with later eras. The word inflation names the era that associates with the third row in the table. Regarding eras that would precede inflation, our modeling points to the possibility for the two eras that the table discusses. Concordance cosmology suggests inflation and the next two eras. Regarding inflation, people hypothesize this era. Possibly, no direct evidence exists for this era. Observations support the notions of the two billions-of-years eras. Concordance cosmology seems to underestimate the rate of separating for the more recent of the two eras. The word speculative denotes that our modeling does not necessarily address the relevant duration or era. The leftmost four columns describe phenomena that our modeling suggests as noteworthy causes for the eras. The construct SOLN abbreviates the word solution. An RDF associates with the PROP solution. Generally, a noteworthy cause associates with notions of acceleration. Generally, an era associates with a range of velocities. A noteworthy cause may gain prominence before an era starts.

\begin{tabular}{|c|c|c|c|c|c|c|}
\hline Force & PROP SOLN & RDF & $\rho_{I}$ & Rate of separating & Duration & Comment \\
\hline Attractive & $2 \mathrm{~g} 1^{6} 2^{6} 3^{6} 8^{6} 16$ & $\Xi^{-6}$ & 6 & Is negative & Speculative & Isomers of $0.5 \mathrm{R}$ and $1 \mathrm{~J}$ form \\
\hline Repulsive & $0 g 1^{6} 3^{6} 4^{6} 8^{6} 16$ & - & 1 & Turns positive & Speculative & Isomers of $0 \mathrm{I}$ form \\
\hline Repulsive & $2 \mathrm{~g} 1^{\prime} 2^{6} 3^{6} 4 \mathrm{x}$ & $\Xi^{-5}$ & 1 & Increases rapidly & Fraction of a second & Inflatons (or, $0 \mathrm{I}$ ) participate \\
\hline Attractive & $2 \mathrm{~g} 1^{\circ} 2^{\circ} 3$ & $\Xi^{-4}$ & 1 & Decreases & Billions of years & Most known particles form \\
\hline Repulsive & $2 \mathrm{~g} 2^{6} 4$ & $\Xi^{-3}$ & 2 & Increases & Billions of years & . \\
\hline Attractive & $2 \mathrm{~g} 2$ & $\Xi^{-2}$ & 6 & Would decrease & & Speculative \\
\hline
\end{tabular}

but just on small (distance) scales. The word free associates with PROP solutions for which $\Sigma \geq 2$ and $8 \notin \Gamma$. Perhaps, a notion of a phase change for the universe - pertains regarding times around inflation.

\subsection{Explanation regarding data about large-scale clumping}

This unit suggests an explanation for the notion that concordance cosmology overestimates largescale clumping of matter.

People suggest that concordance cosmology modeling overestimates large-scale clumping of matter - ordinary matter and dark matter. (For data and discussion, see references [58], [59], [60, and [21].)

We suggest that concordance cosmology modeling associates with a repulsive component - 2(1)g2'4 - of gravity. Our modeling suggests that 2(2)g2'4 pertains. (That is, for each instance of $2 \mathrm{~g}^{\circ} 4$, a reach of two isomers pertains.) The additional (compared to concordance cosmology modeling) repulsion might explain the overestimating - of clumping, per concordance cosmology modeling - that people suggest.

\subsection{Explanation regarding data about interactions between neighboring galaxies}

This unit suggests an explanation for the notion that concordance cosmology might not account for some observations about effects - within individual galaxies - of the gravity associated with nearby galaxies.

People suggest that concordance cosmology modeling might not account for some observations about effects - within individual galaxies - of the gravity associated with nearby galaxies. (For data and discussion, see reference [24].)

We suggest that concordance cosmology modeling associates with a repulsive component - 2(1)g2`4 - of gravity. Our modeling suggests that 2(2)g2'4 pertains. The additional (compared to concordance cosmology modeling) repulsion might explain at least some aspects of the observations that people report.

\subsection{Suggestions regarding galaxy formation}

This unit suggests that our notions regarding long-range interactions and our specifications for dark matter combine to provide insight regarding galaxy formation.

We suggest aspects regarding events leading to the formation of a galaxy.

Reference [35] suggests that galaxies form around early clumps of stuff. The reference associates the word halo with such clumps.

Table 15 suggests that single-isomer stuff - such as stuff that features $0.5 \mathrm{R}$ particles - forms during an era in which the PROP solution $2 \mathrm{~g} 1^{`}{ }^{\circ} 2^{`} 3^{`} 8^{`} 16$ - which associates with attraction - dominates regarding prototype large clumps. Smaller-scale clumps might form before larger-scale clumps. Effects that associate with the PROP solution $2 \mathrm{~g} 1^{\prime}{ }^{\prime}{ }^{`} 3$ - which is attractive might contribute to the formation of smaller-scale clumps. The reach that associates with $2 \mathrm{~g} 1^{\prime} 2^{`} 3$ is one.

We suggest that each one of many early halos associates with one isomer. We associate with such early halos the three-element term one-isomer original clump. We know of no reason why the six isomers would not form such clumps approximately equally. (Concordance cosmology suggests that known elementary fermions form early in the era in which effects that associate with $2 \mathrm{~g}^{\prime}{ }^{\prime} 2^{\prime} 3$ dominate regarding large-scale phenomena. Per remarks above, we suggest that that era starts after the formation of halos. Also, we suggest that our scenario does not depend on whether or when $0.5 \mathrm{~N}$ ' particles first form.)

Table 17 discusses suggestions regarding the formation and early evolution of a galaxy for which a notion of a one-isomer original clump pertains. 
Table 16: Details regarding eras regarding the rate of separating of large clumps. Table 15 discusses the eras. Table 16 de-emphasizes the notion that $0.5 \mathrm{~N}$ ' elementary fermions might form before the beginning of the first multi-billion-years

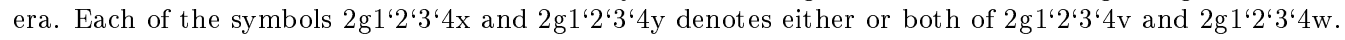

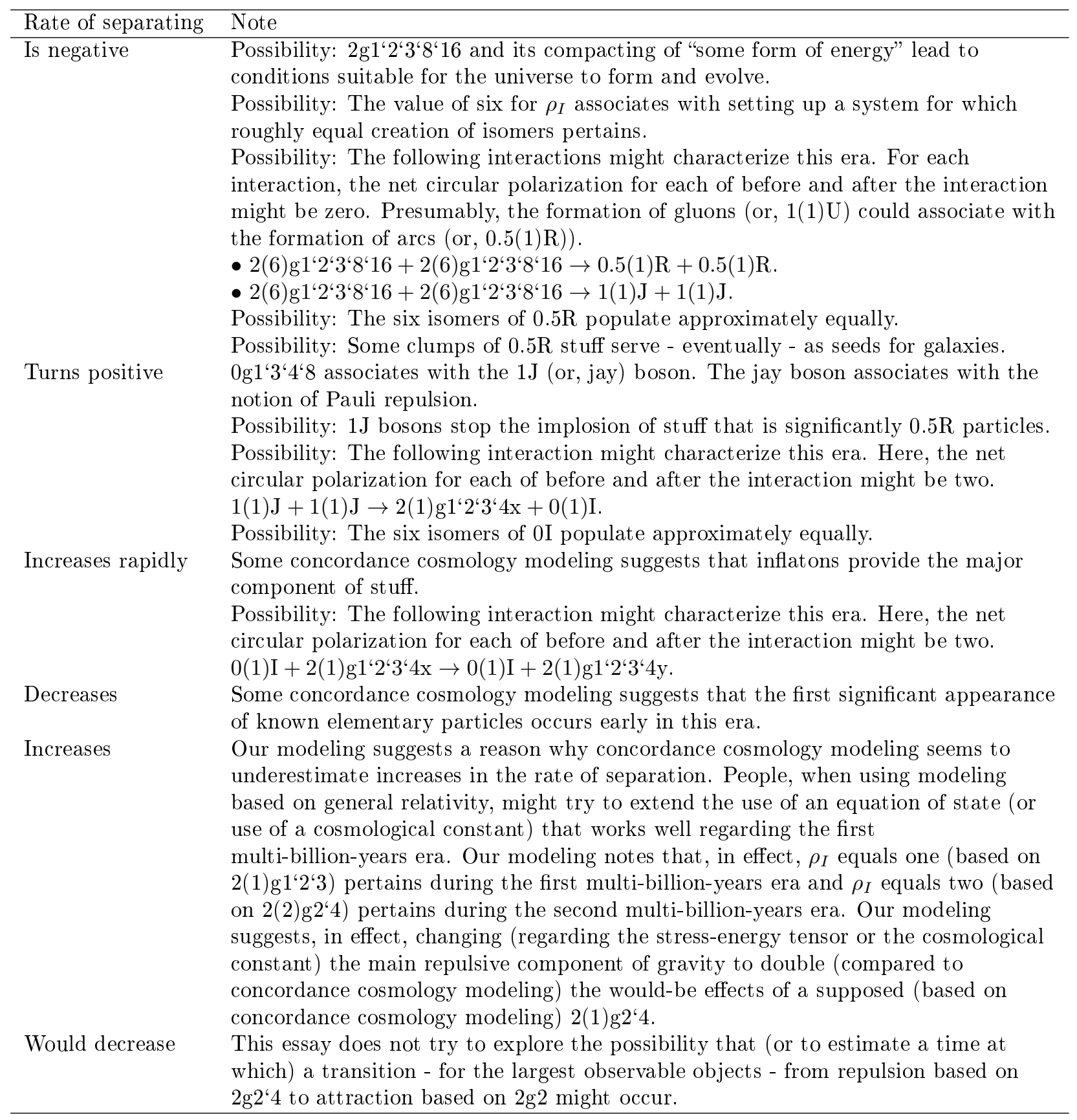

Table 17: Eras and other information regarding the evolution of a galaxy for which a notion of a one-isomer original clump pertains. The table suggests eras, with subsequent rows associating with later eras. The leftmost four columns in the table describe a component of $2 \mathrm{G}$ that is a noteworthy cause for the era. The table associates with a scenario in which a galaxy forms based on one original clump and does not significantly collide with other galaxies. The galaxy might retain some stuff that associates with the repelled isomer.

\begin{tabular}{|c|c|c|c|c|}
\hline Force & PROP solution & RDF & $\rho_{I}$ & Comment \\
\hline Attractive & $2 \mathrm{~g} 1^{6} 2^{6} 3$ & $\Xi^{-4}$ & 1 & A one-isomer original clump forms. \\
\hline Repulsive & $2 \mathrm{~g} 2^{6} 4$ & $\Xi^{-3}$ & 2 & $\begin{array}{l}\text { The original clump repels (some) stuff that associates with the } \\
\text { isomer that associates with the original clump and (most) stuff } \\
\text { that associates with one other isomer. }\end{array}$ \\
\hline Attractive & $2 \mathrm{~g} 2$ & $\Xi^{-2}$ & 6 & $\begin{array}{l}\text { The original clump attracts stuff that associates with the four } \\
\text { not-repelled isomers and stuff that associates with the isomer that } \\
\text { associates with the original clump. }\end{array}$ \\
\hline
\end{tabular}


Presumably, some galaxies form based on two or more clumps, for which all of the clumps associate with just one isomer. Presumably, some galaxies form based on two or more clumps, for which some clumps associate with isomers that are not the same as the isomers that associate with some other clumps.

\subsection{Explanations regarding dark matter data}

This unit shows that our specification for dark matter seems to explain data about galaxies and data about other phenomena.

Table 18 extends aspects of table 7 and provides explanations for data regarding phenomena that might involve dark matter. (Reference [33] influenced our choice of a time range to associate with the word early. References [61] and [62] provide data about collisions. Regarding early galaxies, see table 17. Extrapolating from results that references [35] and 63] discuss regarding ultrafaint dwarf galaxies that orbit the Milky Way galaxy might suggest that the universe contains many DM:OM $1: 0^{+}$ later galaxies. References 64 and 65 provide data and discussion regarding the undetected object.)

\subsection{Explanation regarding data - other than dark matter data - about galaxies}

This unit shows that our specifications for dark matter and for other phenomena might help explain some data - that are not necessarily specific to dark matter - regarding galaxy evolution.

People report the notion that some galaxies seem to stop forming stars. (See reference [66] and reference [67].) Such so-called quenching might take place within three billion years after the Big Bang, might associate with a relative lack of hydrogen atoms, and might pertain to half of a certain type of galaxy. (See reference [67].) Reference [68] discusses a galaxy that seems to have stopped accruing both ordinary matter and dark matter about four billion years after the Big Bang.

We suggest that the quenching and the stopping of accruing nearby matter might associate with repulsion that associates with $2(2) \mathrm{g} 2{ }^{\circ} 4$. Quenching might associate with galaxies for which original clumps featured isomer zero stuff or isomer three stuff. The galaxy that reference [68] discusses might (or might not) associate with the notion of significant presence early on of one of isomers zero and three, one of isomers one and four, and one of isomers two and five. Such early presences might associate with a later lack of nearby stuff for the galaxy to accrue.

\section{Discussion}

This unit discusses some possibilities regarding specific possible elementary particles and regarding dark matter and discusses relationships between our modeling and other modeling.

We associate the two-word term extant modeling with modeling - including Standard Model modeling and concordance cosmology modeling - that other people developed.

\subsection{Possibilities regarding elementary particles}

This unit discusses possibilities regarding properties and existence of hypothesized elementary particles.

This essay seemingly does not suggest an elementary boson that would associate with notions of an axion. Observations that people might associate with effects of axions might associate instead with the difference between our notion of $1(6) \mathrm{g} 1^{\prime} 2^{6} 4$ and extant modeling notions that might associate with notions of $1(1) \mathrm{g} 1^{\prime} 2^{4} 4$.

This essay does not suggest an elementary particle that would associate with notions of a magnetic monopole. Table 1 and table 3 seem not to suggest a $1 \mathrm{G}$ interaction with a monopole other than an electric monopole.

This essay does not necessarily suggest precluding notions of elementary particles that would associate with PROP solutions for which $\log _{4}\left(l_{\max }\right)$ is an integer that exceeds two.

\subsection{Possibilities regarding constraints regarding dark matter}

This unit discusses the extent to which our notion of dark matter comports with constraints about the nature of dark matter - that people associate with data about dark matter or with outputs from extant models that have bases in assumptions about dark matter.

We discuss aspects related to cosmological models.

Reference [35] summarizes some thinking about constraints on dark matter and about notions of dark matter. The article notes that so-called CDM (or, cold dark matter) might comport well with various models. Some models associate with the oneelement term $\Lambda$ CDM. The article notes that people have yet to determine directly whether nature includes CDM stuff. The article notes that people consider that notions of SIDM (or, self-interacting dark matter) might be appropriate regarding nature. People also use other terms, such as the threeword term warm dark matter, to note possible attributes of dark matter. Notions such as SIDM and WDM (or, warm dark matter) arose from modeling that differs from our modeling. We are reluctant to try to closely associate terms such as SIDM or WDM with our modeling. (We suggest that isomer zero $0.5 R$-based stuff, isomer zero $0.5 \mathrm{~N}$ ' stuff, and all stuff associating with isomers one, two, four, and five might comport with some notions of CDM. 
Table 18: Explanations for dark matter ratios and other phenomena. DM:OM denotes a ratio of dark matter effects to ordinary matter effects. Regarding densities of the universe, we posit that DMAI stuff associates with the plus in DM:OM $5^{+}: 1$. Stuff - other than DMAI stuff - that associates with isomers one through five associates with the five in DM:OM $5^{+}: 1$. Regarding some galaxy clusters, we posit that galaxy clusters (that have not collided with other galaxy clusters) associate with DM:OM ratios that are similar to DM:OM ratios for densities of the universe. Regarding some absorption of CMB, we posit that isomer three hydrogen-like atoms account for the half of the absorption for which isomer zero (or, ordinary matter) hydrogen atoms do not account. (See table 4.) The reach of an instance of $1 \mathrm{~g} 3{ }^{6} 4$ is two isomers. (See table 8.) Presumably, the $4 \in \Gamma$ associates with spin and with hyperfine transitions. Regarding galaxies, the notion of early associates with observations that pertain to galaxies that people associate with (or, would, if people could detect the galaxies, associate with) high redshifts. High might associate with $z>7$ and possibly with smaller values of $z$. Here, $z$ denotes redshift. Regarding galaxies for which the DM:OM is $5^{+}: 1$, DMAI stuff associates with the plus in DM:OM $5^{+}: 1$. Regarding the undetected object, we posit that the undetected object is a clump of dark matter. Data regarding stellar stream GD-1 suggest the possibility of effects from a yet-to-be-detected non-ordinary-matter clump with a mass of $10^{6}$ to $10^{8}$ solar masses.

\begin{tabular}{|c|c|}
\hline Aspect & Comment \\
\hline DM:OM $5^{+}: 1-$ Densities of the universe & - \\
\hline DM:OM $5^{+}: 1$ - Some galaxy clusters & - \\
\hline DM:OM $1: 1$ - Some absorption of CMB & Half of the absorption might be via DM. \\
\hline DM:OM $0^{+}: 1$ - Some early galaxies & $\begin{array}{l}\text { For each of some early galaxies, each original clump associates } \\
\text { with isomer zero. Later, the galaxy might accumulate DM. }\end{array}$ \\
\hline DM:OM $0^{+}: 1$ - Some later galaxies & $\begin{array}{l}\text { Some early } \mathrm{DM}: \mathrm{OM} 0^{+}: 1 \text { galaxies survive (without } \\
\text { significant collisions with galaxies for which } \mathrm{DM}: \mathrm{OM} \text { is not } \\
0^{+}: 1 \text { ) until later times. }\end{array}$ \\
\hline DM:OM $1: 0^{+}$- Some early galaxies & $\begin{array}{l}\text { For each of some early galaxies, each original clump associates } \\
\text { with an isomer other than isomer zero. Early on, the density } \\
\text { of OM stars is small and people do not detect the galaxy. } \\
\text { Later, the galaxy might accumulate enough OM to be visible. }\end{array}$ \\
\hline DM:OM $1: 0^{+}$- Some later galaxies & $\begin{array}{l}\text { Some early DM:OM } 1: 0^{+} \text {galaxies survive (without } \\
\text { significant collisions with galaxies for which the DM:OM is not } \\
1: 0^{+} \text {) until later times. }\end{array}$ \\
\hline DM:OM $\sim 4: 1$ - Some later galaxies & $\begin{array}{l}\text { An original clump might associate with any isomer other than } \\
\text { isomer three. (Isomer three repels OM stuff.) Eventually, the } \\
\text { galaxy accumulates enough stuff (that does not associate with } \\
\text { the isomer that associates with the original clump) to have a } \\
\text { DM:OM ratio that is somewhat near } 4: 1 \text {. }\end{array}$ \\
\hline DM:OM $5^{+}: 1$ - Many later galaxies & $\begin{array}{l}\text { Over time, galaxies collide. Collisions tend to result in the } \\
\text { formation of larger galaxies that include much stuff from } \\
\text { smaller galaxies. A later galaxy that results from enough } \\
\text { collisions is likely to associate with somewhat similar - across } \\
\text { the six isomers - amounts of stuff from originally one- (or few-) } \\
\text { isomer original clump galaxies. }\end{array}$ \\
\hline Undetected object in the Milky Way galaxy & The object might be DM. \\
\hline
\end{tabular}


We suggest that the remaining dark matter stuff or, isomer three OMSE stuff - might associate with some notions of WDM and with some notions of SIDM.)

We suggest that our notion of dark matter is not necessarily incompatible with constraints - that have bases in cosmological models - on dark matter.

We discuss aspects related to collisions of pairs of galaxy clusters. In particular we discuss the Bullet Cluster collision of two galaxy clusters. (Reference 69] discusses the Bullet Cluster.) Presumably, observations regarding other such collisions might pertain.

Observations suggest two general types of trajectories for stuff. Most dark matter - from either one of the clusters - exits the collision with trajectories consistent with having interacted just gravitationally with the other cluster. Also, ordinary matter stars - from the same cluster - exit the collision with trajectories consistent with having interacted just gravitationally with the other cluster. However, ordinary matter IGM (or, intergalactic medium) - from the one cluster - lags behind the cluster's ordinary matter stars and dark matter. That ordinary matter IGM interacted electromagnetically with the other cluster's ordinary matter IGM, as well as gravitationally with the other cluster.

We are uncertain as to the extent to which observational data suggests that essentially no dark matter lags the bulk of dark matter. Should the fraction of lagging dark matter be too small, we might need to reconsider the extent to which isomer three differs from isomer one. For one example, possibly isomer three has right-handed elementary fermions but interactions involving such fermions model as retaining aspects of left-handedcentric interactions that associate with isomer zero. For another example, possibly isomer three does not evolve adequately similarly to isomer zero. To the extent that isomer three adequately differs from or does not evolve similarly to isomer zero, our explanation regarding CMB depletion via - in part - interactions with dark matter hydrogen-like atoms might be inaccurate (for example, based on an inaccurate estimate of the number of isomer three hydrogenlike atoms).

We suggest that our notion of dark matter is not necessarily incompatible with constraints - that have bases in observations of collisions of galaxy clusters - on dark matter.

\subsection{Possibilities regarding interactions involving the jay boson}

This unit discusses interactions that involve jay bosons.

\subsubsection{We discuss interactions - that involve jay bosons - that might take place before or dur- ing inflation.}

We consider interactions in which two jay bosons move in parallel, interact, and produce one aye boson plus something else. Here, we assume that conservation of angular momentum pertains and that one can de-emphasize orbital angular momentum. We consider two cases. In the first case, the two jay bosons have the same (one of either right or left) circular polarization. Conservation of angular momentum allows an outgoing combination of one $2 \mathrm{G}$ particle and one $0 \mathrm{I}$ particle. Conservation of angular momentum precludes producing one $1 \mathrm{G}$ particle and one 0I particle. In the second case, one jay boson has left circular polarization and the other jay boson has right circular polarization. Conservation of angular momentum allows the production of two $0 \mathrm{I}$ particles and prohibits the production of one $1 \mathrm{G}$ particle and one $0 \mathrm{I}$ particle.

The two cases might comport with notions that gravitation can be significant during inflation and that electromagnetism might become significant essentially only after inflation. The three cases might comport with the notion that jay bosons form before aye bosons form. (See table 15.)

\subsubsection{We discuss so-called Pauli crystals.}

Reference 70 and reference 71 report detection of Pauli crystals. We suggest that modeling based on the notion of jay bosons might help explain relevant phenomena.

\subsubsection{We discuss the notion of Pauli repulsion.}

Extant modeling includes the notion that two identical fermions cannot occupy the same state. Regarding extant modeling, one notion is that repulsion between identical fermions associates with overlaps of wave functions. Another notion features wave functions that are anti-symmetric with respect to the exchange of two identical fermions.

Our modeling might be compatible with such aspects of extant modeling and, yet, not necessitate for kinematics modeling - the use of wave functions. Modeling based on jay bosons might suffice.

Modeling based on jay bosons might suggest that prevention of two identical fermions from occupying the same state might associate with, in effect, trying to change aspects related to the fermions. Notions of changing a spin orientation or, for elementary fermions, changing a flavour might pertain.

\subsubsection{We discuss a possible discrepancy-regarding energy levels in positronium - between extant modeling and observation.}

Reference 72 and reference 73 discuss the transition - between two states of positronium - 
characterized by the expression $2^{3} S_{1} \rightarrow 2^{3} P_{0}$. People discuss the energy that associates with the transition. Four standard deviations below the nominal observed value of energy approximately equals four standard deviations above the nominal value of energy that extant modeling suggests.

Perhaps, notions regarding jay bosons extend to explain the might-be discrepancy regarding positronium. (For example, thinking of extant modeling based on the Dirac equation, a notion of virtual charge exchange or virtual flavour change might pertain.)

To the extent that extant modeling does not suffice, modeling related to the jay boson might close the gap between observation and modeling.

\subsection{Possibilities regarding right-handed $W$ bosons}

This unit discusses the possibility that our modeling suggests approximately the fraction of $\mathrm{W}$ bosons that are right-handed that result from decays of top quarks.

\subsubsection{We consider the notion that some values of calculated non-inertial mass-like quantities have physics significance.}

Aspects related to table 12 , table 13 , and table 14 suggest values of calculated masses that do not associate with masses of known or suggested elementary particles. For example, our modeling does not suggest that $m(5,3)$ associates with the inertial mass of an isomer one charged lepton. However, perhaps such mass-like quantities associate with some measurable aspects of nature. For charged leptons and $0 \leq l \leq 4$ and $0 \leq l_{2} \leq 2$, $m\left(3(l+1)+l_{2}, 3\right)=\beta m\left(3(l+0)+l_{2}, 3\right)$. One might conjecture that isomer zero observations of some aspects of isomer one phenomena associate with notions of non-inertial mass-like quantities that are $\beta$ times the inertial masses for isomer zero elementary particles (and that are $\beta$ times inertial masses for the counterpart isomer one elementary particles).

Furthermore, isomer one might associate with right-handedness in a manner similar to the association of isomer zero with left-handedness. (See discussion related to table 14.)

\subsubsection{We develop modeling that might associate with the fraction of right-handed $W$ bosons that one type of quark decay might produce.}

Reference 74 discusses the fraction of decays - of ordinary matter top quarks for which the decay products include $\mathrm{W}$ bosons - that might produce right-handed $\mathrm{W}$ bosons. The fraction, $f_{+}$, is $3.6 \times 10^{-4}$. Reference $[8]$ provides a confidence level of 90 percent that the rest energy of a $W_{R}$ (or, righthanded $\mathrm{W}$ boson) exceeds $715 \mathrm{GeV}$. (Perhaps, note also, reference [75].)
Based on notions of scaling that might calculate non-inertial mass-like quantities, one might conjecture that our modeling suggests that $f_{+} \sim$ $e^{\left(\beta^{-1}\right)}-1 \approx \beta^{-1} \approx 2.9 \times 10^{-4}$. This estimate might not be incompatible with results that reference [74] discusses. A notion of $m_{\text {non-inertial, }} \mathrm{W}_{R}$ isomer one $c^{2}=$ $\beta m_{\mathrm{W}} c^{2} \approx 2.8 \times 10^{5} \mathrm{GeV}$ might pertain. Here, the notion of non-inertial mass-like quantity might associate with inferences that associate with $1 \mathrm{G}$ or $1 \mathrm{~W}$ and do not associate directly with $2 \mathrm{G}$.

\subsection{Relationships between our modeling and other modeling}

This unit discusses relationships between our modeling and types of extant models.

\subsubsection{We suggest perspective about our modeling.}

Our modeling features two bases.

One basis unifies and decomposes aspects of electromagnetism and gravity. For each of electromagnetism and gravity, the decomposition seems to associate well with properties - of objects - that people can measure and that extant modeling features. For electromagnetism, the properties include charge and magnetic moment. For gravity, the properties include mass and moments of inertia.

One basis features isomers of elementary particles that do not intermediate long-range interactions and features instances of components of longrange interactions.

Our modeling extends from the two bases to do the following. Match all known elementary particles and suggest possible other elementary particles. Describe dark matter. Point to explanations for data that extant modeling seems not to explain. Suggest data that might associate with future observations.

We suggest the possibility that the notion that our work explains phenomena that extant modeling does not explain points to usefulness for our work. Some explanations have quantitative bases but - to the extent that this essay uses the explanations are qualitative. Presumably, people can use simulations to help verify or refute some of our qualitative explanations. At least one explanation - regarding depletion of CMB - is quantitative. Generally, we know of no cases in which our suggestions that address possible gaps between extant modeling and observations point - compared to extant modeling in a wrong direction regarding closing gaps.

We suggest the possibility that the notion that our work suggests specifications and data that extant modeling does not suggest points to possible usefulness for our work. Our suggestions include a specification for dark matter, specifications for new elementary particles, and more (than current measurements provide) accurate masses for neutrinos and some other known elementary particles. 
We suggest that the small set of bases for our modeling, the breadth of seemingly coherent scope of our modeling, the simplicity of relevant Diophantine equations, and the possible ease of integrating our modeling and extant modeling point to possible usefulness for our work.

\subsubsection{We suggest approximate relationships be- tween aspects of our modeling and types of kinematics models.}

Table 19 discusses approximate relationships between aspects of our modeling and types of kinematics models.

\subsubsection{We develop modeling that might estimate the anomalous magnetic moment for the tau el- ementary particle.}

We discuss the case of QED. In particular, we explore modeling regarding anomalous magnetic moments for $0.5 \mathrm{C}$ elementary particles (or, charged leptons).

Table 4 associates two CURR solutions with the relevant (or, $3 g 1^{6} 2$ ) PROP solution. The $3 g 1^{6} 6{ }^{6} 8$ CURR solution includes 6 in $\Gamma$. We posit that the strength of $3 \mathrm{~g} 1^{6} 6^{6} 8$ can vary based on mass, but not based on charge. The $3 \mathrm{~g} 2^{6} 7^{6} 8$ CURR solution includes 7 in $\Gamma$. We posit that the strength of $3 \mathrm{~g} 2^{6} 7^{6} 8$ can vary based on charge, but not based on mass.

We explore the notion that one can express $a_{c l}$, the anomalous magnetic moment for the $c l$ charged lepton, via the expression $a_{7}+a_{6} t_{c l}$. Here, $a_{7}$ might vary only with charge and would be a constant with respect to a choice between $c l=e$ (for the electron), $c l=\mu$ (for the muon), and $c l=\tau$ (for the tau). Here, $a_{6}$ might vary only with mass. We assume that $t_{\mathrm{cl}}$ is $\left(\log \left(m_{\mathrm{cl}} / m_{e}\right)\right)^{2}$. (Perhaps, compare with table 11 and with aspects - that comport with squares of properties - of table 12 . The notion of squares of properties might associate with notions of self-interactions.) Based on data that reference [8] provides regarding the electron and the muon, we calculate $a_{7}$ and $a_{6}$. Then, we calculate a value, $a_{\tau, \mathrm{PM}}$, for $a_{\tau}$. Here, PM denotes the two-word term proposed modeling. PM associates with our work. Reference [76] provides, based on Standard Model modeling techniques, a first-order result - which we call $a_{\tau, \mathrm{SM}}-$ for $a_{\tau}$. Here, SM denotes the two-word term Standard Model. The value of $a_{\tau, \mathrm{PM}}$ results in a value of $\left(a_{\tau, \mathrm{PM}}-a_{\tau, \mathrm{SM}}\right) / a_{\tau, \mathrm{SM}}$ of approximately -0.00228 . Each of $a_{\tau, \mathrm{PM}}$ and $a_{\tau, \mathrm{SM}}$ comports with experimental data that reference [8] provides.

\subsubsection{We discuss aspects related to the value of two for reach (or, $\rho_{I}$ ).}

This essay suggests that $\rho_{I}=2$ pertains for some components of long-range interactions (or, LRI). This essay suggests that the notion of $\rho_{I}=2$ might have importance regarding explaining data regarding the following - some depletion of $\mathrm{CMB}$, large-scale clumping, the recent multi-billion-years era of increases regarding the rate of separation of large clumps, gravitational interactions between neighboring galaxies, and galaxy formation.

\section{Conclusions}

Our work suggests augmentations - to physics modeling - that produce results that may provide progress regarding the following physics opportunities. Complete the list of elementary particles. Describe dark matter. Explain ratios of dark matter to ordinary matter. Explain eras in the history of the universe. Link properties of objects. Interrelate physics models.

We use our modeling to match data that other modeling matches.

We use our modeling to suggest explanations for data that other modeling seems not to explain.

We use our modeling to suggest results regarding data that people have yet to gather.

The breadth and depth of the matched data might suffice to justify using our modeling.

The breadth and unity - within itself and with physics modeling that people use successfully - of our modeling might support the usefulness of our modeling.

\section{Acknowledgments}

The following people pointed, via personal contact, to topics or aspects that we considered for inclusion in the scope of our work: Andrea Albert, Raphael Bousso, Lance Dixon, Persis Drell, Immanuel Freedman, Ervin Goldfain, Kamal Melek Hanna, Wick Haxton, Nick Hutzler, William Lama, Surhud More, Holger Muller, J. Xavier Prochaska, Martin Rees, Harrison Rose, and Mak Tafazoli.

The following people provided comments regarding the effectiveness of drafts that led to parts of this essay: Immanuel Freedman, Ervin Goldfain, Vesselin Gueorguiev, William Lama, Tom Lawrence, and Mak Tafazoli.

The following people helped publish aspects leading to work that this essay describes: Charles K. Chui, Kamal Melek Hanna, Keith Jones, and Zeger Karssen. (Perhaps, note reference [77] and reference [78.) The following people provided or pointed to aspects regarding expressing or propagating the work: Elliott Bloom, Man Ho Chan, Maxwell Chertok, Charles K. Chui, Andrei Lucian Dragoi, Steven Frautschi, Carl Frederick, Ervin Goldfain, Vesselin Gueorguiev, Ioannis Haranas, Richard B. Holmes, Frank Hiroshi Ling, Michael Mulhearn, Richard A. Muller, Stephen Perrenod, Paul Preuss, Amal Pushp, Amir Sharif, and Wendy Shi. The following people suggested perspective, 
Table 19: Approximate relationships between aspects of our modeling and types of kinematics models. $k_{I}$ denotes a number - one or six - of isomers. Extant modeling associates with $k_{I}=1$. Each of some of the items in the symbol column does not associate with an extant modeling notion or symbol. Regarding NEW, the row in the table assumes that electromagnetism is not relevant. Regarding EAM, $1 \mathrm{~g} 1$ associates with non-moving charge and 1g7' 8 associates with moving charge. No other components have relevance. This modeling associates with charge-current 4-vectors and with Maxwell's equations. Regarding GNR, the notions that $l_{\max }$ PROP is 4 (or is 16 ) and $l_{\max }$ CURR is 8 (or is 32 ) might not pertain exactly regarding general relativity. Also regarding GNR and the possible case of $k_{I}=6$, the notion of geodesic motion would not necessarily pertain. For example, consider an isomer zero star and three possible planets. The planets are identical except that one planet associates with isomer zero, one planet associates with isomer one, and one planet associates with isomer three. The planets start out on identical orbits. We consider six cases. First assume that - out of the $2 \mathrm{~g}^{\prime}$ components only 2(6)g2 pertains. The planets traverse identical orbits. Second, assume that 2(2)g2" 4 associates with nonzero effects. The isomer one planet orbits as if $2 \mathrm{~g}^{\star} 4$ does not pertain. The isomer zero planet and the isomer three planet traverse a trajectory that differs from the trajectory that is common for the previous four cases. QCD associates with $1 \mathrm{U}, 0.5 \mathrm{Q}_{1 / 3}$, and $0.5 \mathrm{Q}_{2 / 3}$. We suggest the possibility that QCD also associates with $0.5 \mathrm{R}$. WIP associates with $1 \mathrm{~W}$ and $1 \mathrm{Z}$. PEP associates with $1 \mathrm{~J}$, each $0.5 \Phi$ family, and fermions that are not elementary particles.

\begin{tabular}{llllll}
\hline Kinematics modeling & Range of $\Sigma$ & $l_{\max }$ PROP & $l_{\max }$ CURR & $k_{I}$ & Symbol \\
\hline Newtonian gravity & 2 & 2 & 2 & 1 & NEW \\
Special relativity & 1,2 & 2 & 8 & 1 & SPR \\
Electrostatics & 1 & 1 & 1 & 1 & EST \\
Electromagnetism & 1 & 1 & 8 & 1 & EAM \\
Quantum electrodynamics & 1,3 & 2 & 8 & 1 & QED \\
General relativity & 2 & 4 (or 16$)$ & 8 (or 32$)$ & 1 & GNR \\
Quantum chromodynamics & 0 & 16 & 32 & 1 & QCD \\
Weak-interaction phenomena & 0 & 4 & 8 & 1 & WIP \\
Pauli repulsion phenomena & 0 & 16 & 32 & 1 & PEP \\
Suggested by our modeling & $0,1,2,3,4$ & 16 & 32 & 6 & PRM \\
\hline
\end{tabular}

means, or suggestions regarding people with whom to try to have discussions: Vint Cerf, Yanbei Chen, James S. Clegg, Bill Daul, Jiggs Davis, George Djorgovski, Erica Ellingson, Ron Fredericks, Vesselin Gueorguiev, Tucker Hiatt, William Lama, Lianne La Reine, Robert Morgan, Doug Osheroff, Kennan Salinero, Jim Spohrer, Peter Walstrom, and Jon F. Wilkins.

\section{Funding statement}

This research did not receive any specific grant from funding agencies in the public, commercial, or not-for-profit sectors.

\section{Conflicts of interest}

The author declares no conflict of interest.

\section{References}

[1] Jairzinho Ramos Medina. Gravitoelectromagnetism (GEM): A Group Theoretical Approach. PhD thesis, Drexel University, August 2006. Link: https://core.ac.uk/download/pdf/190333514.pdf. 1.3, 2

[2] David Delphenich. Pre-Metric Electromagnetism as a Path to Unification. In Unified Field Mechanics. World Scientific, September 2015 . Link: https://arxiv.org/ftp/arxiv/papers/1512/1512.05183.pdf. 1.3, 2

[3] S. Gasiorowicz and P. Langacker. Elementary Particles in Physics. University of Pennsylvania. Link: https://www.physics.upenn.edu/pgl/e27/E27.pdf. 1.4.1

[4] A. Hebecker and J. Hisano. 94: Grand Unified Theories. In P. A. Zyla and others (Particle Data Group), Prog. Theor. Exp. Phys, 083C01 (2020) and 2021 update, 2019. Link: https://pdg.lbl.gov/2021/reviews/rpp2020-rev-guts.pdf. 1.4.1

[5] A. Ringwald, L. J. Rosenberg, and G. Rybka. 91: Axions and Other Similar Particles. In P. A. Zyla and others (Particle Data Group), Prog. Theor. Exp. Phys, 083 C01 (2020) and 2021 update, 2019. Link: https://pdg.lbl.gov/2021/web/viewer.html?file=1.4.1

[6] S. Rolli and M. Tanabashi. 95: Leptoquarks. In P. A. Zyla and others (Particle data Group), Prog. Theor. Exp. Phys, $083 \mathrm{C01}$ (2020) and 2021 update, 2019. Link: https://pdg.lbl.gov/2021/web/viewer.html?file=1.4.1 
[7] D. Milstead and E. J. Weinberg. 96: Magnetic Monopoles. In P. A. Zyla and others (Particle Data Group), Prog. Theor. Exp. Phys, 083C01 (2020) and 2021 update, 2019. Link: https://pdg.lbl.gov/2021/web/viewer.html?file=1.4.1

[8] P. A. Zyla et al. Review of Particle Physics. PTEP, 2020(8):083C01, 2020. Link: https://pdg.lbl.gov/2020/citation.html. 1.4.1, 2.3.1, 3.1.2, 3.1.2, 3.1.2, 3.1.3, 3.1.3, 4.4.2, 4.5.3

[9] Lotty Ackerman, Matthew R. Buckley, Sean M. Carroll, and Marc Kamionkowski. Dark matter and dark radiation. Physical Review D, 79:023519, January 2009. Link: https://link.aps.org/doi/10.1103/PhysRevD.79.023519. 1.4.1

[10] Brian Green. Until the End of Time: Mind, Matter, and Our Search for Meaning in an Evolving Universe. Alfred A. Knopf, February 2020. Link: https://www.penguinrandomhouse.com/books/549600/until-the-end-of-time-by-brian-greene/. $1.4 .1,2.2 .1,3.3 .3$

[11] Charles W. Misner, Kip S. Thorne, and John Archibald Wheeler. Gravitation. University of Princeton Press, October $2017 . \quad$ Link: https://press.princeton.edu/books/hardcover/9780691177793/gravitation. 1.4.1

[12] M. C. Gonzalez-Garcia and M. Yokoyama. 14: Neutrino Masses, Mixing, and Oscillations. In P. A. Zyla and others (Particle Data Group), Prog. Theor. Exp. Phys, 083C01 (2020) and 2021 update, 2019. Link: https://pdg.lbl.gov/2021/reviews/rpp2020-rev-neutrino-mixing.pdf. 1.4.1

[13] T. Damour. 21: Experimental Tests of Gravitational Theory. In P. A. Zla and others (Particle Data Group), Prog. Theor. Exp. Phys, 083C01 (2020) and 2021 update, 2019. Link: https://pdg.lbl.gov/2021/reviews/rpp2020-rev-gravity-tests.pdf. 1.4 .1

[14] M. Kramer, I.H. Stairs, R.N. Manchester, N. Wex, A.T. Deller, W.A. Coles, M. Ali, M. Burgay, F. Camilo, I. Cognard, et al. Strong-Field Gravity Tests with the Double Pulsar. Phys. Rev. X, 11(4):041050, December 2021. Link: https://journals.aps.org/prx/abstract/10.1103/PhysRevX.11.041050. 1.4.1

[15] K. A. Olive and J. A. Peacock. 22: Big-Bang Cosmology. In P. A. Zyla and others (Particle Data Group), Prog. Theor. Exp. Phys, 083C01 (2020) and 2021 update, 2019. Link: https://pdg.lbl.gov/2021/web/viewer.html?file=1.4.2

[16] J. Ellis and D. Wands. 23: Inflation. In P. A. Zyla and others (Particle Data Group), Prog. Theor. Exp. Phys, $083 \mathrm{C} 01$ (2020) and 2021 update, 2019. Link: https://pdg.lbl.gov/2021/web/viewer.html?file=1.4.2

[17] D. H. Weinberg and M. White. 28: Dark Energy. In P. A. Zyla and others (Particle Data Group), Prog. Theor. Exp. Phys, 083C01 (2020) and 2021 update, 2019. Link: https://pdg.lbl.gov/2021/reviews/rpp2020-rev-dark-energy.pdf. 1.4 .2

[18] L. Verde, T. Treu, and A. G. Riess. Tensions between the early and late Universe. Nature Astronomy, 3(10):891-895, September 2019. Link: https://www.nature.com/articles/s41550-019-0902-0. 1.4.2, 3.3.3

[19] Johanna L. Miller. Gravitational-lensing measurements push Hubble-constant discrepancy past 5\%. Physics Today, 2020(1):0210a, February 2020. Link: https://physicstoday.scitation.org/do/10.1063/PT.6.1.20200210a/full/. 1.4.2, 3.3.3

[20] Thomas Lewton. What Might Be Speeding Up the Universe's Expansion? Quanta Magazizne, May 2020. Link: https://www.quantamagazine.org/why-is-the-universe-expanding-so-fast$20200427 / .1 .4 .2,3.3 .3$

[21] Christopher Wanjek. Dark Matter Appears to be a Smooth Operator. Mercury, 49(3):1011, October 2020. Link: https://astrosociety.org/news-publications/mercury-online/mercuryonline.html/article/2020/12/10/dark-matter-appears-to-be-a-smooth-operator. 1.4.2, 3.3.3, 3.4

[22] L. Baudis and S. Profumo. 27: Dark Matter. In P. A. Zyla and others (Particle Data Group), Prog. Theor. Exp. Phys, 083C01 (2020) and 2021 update, 2019. Link: https://pdg.lbl.gov/2021/reviews/rpp2020-rev-dark-matter.pdf. 1.4 .3 
[23] Houjun Mo, Frank van den Bosch, and Simon White. Galaxy Formation and Evolution. Cambridge University Press, Cambridge, UK, 2010. Link: https://www.cambridge.org/us/academic/subjects/physics/astrophysics/galaxy-formation-andevolution-1. 1.4.3

[24] Kyu-Hyun Chae, Federico Lelli, Harry Desmond, Stacy S. McGaugh, Pengfei Li, and James M. Schombert. Testing the Strong Equivalence Principle: Detection of the External Field Effect in Rotationally Supported Galaxies. The Astrophysical Journal, 904(1):51, November 2020. Link: https://iopscience.iop.org/article/10.3847/1538-4357/abbb96/meta. 1.4.3, 3.5

[25] Jean-Pierre Amiet and Stefan Weigert. Commensurate harmonic oscillators: Classical symmetries. Journal of Mathematical Physics, 43(8):4110-4126, August 2002. Link: https://sites.ifi.unicamp.br/aguiar/files/2014/10/P034ClassCommensurateOscillators2002.pdf. 2.1 .2

[26] Ewa L. Lokas and Gary A. Mamon. Dark matter distribution in the Coma cluster from galaxy kinematics: breaking the mass-anisotropy degeneracy. Monthly Notices of The Royal Astronomical Society, 343(2):401-412, August $2003 . \quad$ Link: https://academic.oup.com/mnras/article/343/2/401/1038976. 2.3.1

[27] Elena Rasia, Giuseppe Tormen, and Lauro Moscardini. A dynamical model for the distribution of dark matter and gas in galaxy clusters. Monthly Notices of The Royal Astronomical Society, 351(1):237-252, June 2004. Link: https://academic.oup.com/mnras/article/351/1/237/1004623. 2.3.1

[28] Lawrence Rudnick. The Stormy Life of Galaxy Clusters: astro version. January 2019. Link: https://ned.ipac.caltech.edu/level5/March19/Rudnick/frames.html. 2.3.1

[29] Lawrence Rudnick. The stormy life of galaxy clusters. Physics Today, 72(1):46-52, January 2019. Link: https://physicstoday.scitation.org/doi/full/10.1063/PT.3.4112. 2.3.1

[30] Judd D. Bowman, Alan E. E. Rogers, Raul A. Monsalve, Thomas J. Mozdzen, and Nivedita Mahesh. An absorption profile centred at 78 megahertz in the sky-averaged spectrum. Nature, 555(7694):6770, March 2018. Link: https://www.nature.com/articles/nature25792. 2.3.1

[31] Rennan Barkana. Possible interaction between baryons and dark-matter particles revealed by the first stars. Nature, 555(7694):71-74, March 2018. Link: https://www.nature.com/articles/nature25791. 2.3.1

[32] Paolo Panci. 21-cm line Anomaly: A brief Status. In 33rd Rencontres de Physique de La Vallee d'Aoste, July 2019. Link: https://cds.cern.ch/record/2688533. 2.3.1

[33] Peter Behroozi, Risa Wechsler, Andrew Hearin, and Charlie Conroy. UniverseMachine: The correlation between galaxy growth and dark matter halo assembly from z $=0-10$. Monthly Notices of The Royal Astronomical Society, 488(3):3143-3194, May 2019. Link: https://academic.oup.com/mnras/article/488/3/3143/5484868. 2.3.1, 3.7

[34] R. Genzel, N. M. Forster Schreiber, H. Ubler, P. Lang, T. Naab, R. Bender, L. J. Tacconi, E. Wisnioski, S. Wuyts, T. Alexander, et al. Strongly baryon-dominated disk galaxies at the peak of galaxy formation ten billion years ago. Nature, 543(7645):397-401, March 2017. Link: https://www.nature.com/articles/nature21685. 2.3.1

[35] Joshua D. Simon and Marla Geha. Illuminating the darkest galaxies. Physics Today, 74(11):30-36, November 2021. Link: https://physicstoday.scitation.org/doi/10.1063/PT.3.4879. 2.3.1, 3.6, 3.7, 4.2

[36] J. Jimenez-Vicente, E. Mediavilla, C. S. Kochanek, and J. A. Munoz. Dark Matter Mass Fraction in Lens Galaxies: New Estimates from Microlensing. Astrophysical Journal, 799(2):149, January 2015. Link: http://stacks.iop.org/0004-637X/799/i=2/a=149. 2.3.1

[37] J. Jimenez-Vicente, E. Mediavilla, J. A. Munoz, and C. S. Kochanek. A Robust Determination of the Size of Quasar Accretion Disks Using Gravitational Microlensing. Astrophysical Journal, 751(2):106, May 2012. Link: https://iopscience.iop.org/article/10.1088/0004-637X/751/2/106. 2.3.1 
[38] Pieter van Dokkum, Roberto Abraham, Jean Brodie, Charlie Conroy, Shany Danieli, Allison Merritt, Lamiya Mowla, Aaron Romanowsky, and Jielai Zhang. A High Stellar Velocity Dispersion and 100 Globular Clusters for the Ultra-diffuse Galaxy Dragonfly 44. Astrophysical Journal, 828(1):L6, August 2016. Link: http://iopscience.iop.org/article/10.3847/2041-8205/828/1/L6. 2.3.1

[39] Shannon Hall. Ghost galaxy is 99.99 per cent dark matter with almost no stars. New Scientist, August 2016. Link: https://www.newscientist.com/article/2102584-ghost-galaxy-is-99-99-per-centdark-matter-with-almost-no-stars / . 2.3.1

[40] Pavel E. Mancera Pina, Filippo Fraternali, Elizabeth A. K. Adams, Antonino Marasco, Tom Oosterloo, Kyle A. Oman, Lukas Leisman, Enrico M. di Teodoro, Lorenzo Posti, Michael Battipaglia, et al. Off the Baryonic Tully-Fisher Relation: A Population of Baryondominated Ultra-diffuse Galaxies. Astrophysical Journal, 883(2):L33, September 2019. Link: https://iopscience.iop.org/article/10.3847/2041-8213/ab40c7/meta. 2.3 .1

[41] Pavel E. Mancera Pina, Filippo Fraternali, Tom Oosterloo, Elizabeth A. K. Adams, Kyle A. Oman, and Lukas Leisman. No need for dark matter: resolved kinematics of the ultra-diffuse galaxy AGC 114905. Mon. Not. R. Astron Soc., December 2021. Link: https://academic.oup.com/mnras/advance-article/doi/10.1093/mnras/stab3491/6461100. 2.3.1

[42] Qi Guo, Huijie Hu, Zheng Zheng, Shihong Liao, Wei Du, Shude Mao, Linhua Jiang, Jing Wang, Yingjie Peng, Liang Gao, et al. Further evidence for a population of darkmatter-deficient dwarf galaxies. Nature Astronomy, 4(3):246-251, November 2019. Link: https://www.nature.com/articles/s41550-019-0930-9. 2.3.1

[43] Pieter van Dokkum, Shany Danieli, Roberto Abraham, Charlie Conroy, and Aaron J. Romanowsky. A Second Galaxy Missing Dark Matter in the NGC 1052 Group. Astrophysical Journal, 874(1):L5, March 2019. Link: https://iopscience.iop.org/article/10.3847/2041-8213/ab0d92. 2.3.1

[44] Charles Day. A primordial merger of galactic building blocks. Physics Today, 2021(1):0614a, June 2021. Link: https://physicstoday.scitation.org/do/10.1063/PT.6.1.20210614a/full/. 2.3.1

[45] Yuta Tarumi, Naoki Yoshida, and Anna Frebel. Formation of an Extended Stellar Halo around an Ultra-faint Dwarf Galaxy Following One of the Earliest Mergers from Galactic Building Blocks. The Astrophysical Journal Letters, 914(1):L10, June 2021. Link: https://iopscience.iop.org/article/10.3847/2041-8213/ac024e. 2.3.1

[46] Massimo Meneghetti, Guido Davoli, Pietro Bergamini, Piero Rosati, Priyamvada Natarajan, Carlo Giocoli, Gabriel B. Caminha, R. Benton Metcalf, Elena Rasia, Stefano Borgani, et al. An excess of small-scale gravitational lenses observed in galaxy clusters. Science, 369(6509):1347-1351, September 2020. Link: https://science.sciencemag.org/content/369/6509/1347. 2.3.1

[47] Maria Temming. Dark matter clumps in galaxy clusters bend light surprisingly well. Science News, September 2020. Link: https://www.sciencenews.org/article/dark-matter-clumps-galaxy-clustersbend-light-surprisingly-well. 2.3.1

[48] P. Vogel and A. Piepke. Neutrino Properties. In P. A. Zyla and others (Particle Data Group), Prog. Theor. Exp. Phys, $083 \mathrm{C} 01$ (2020) and 2021 update, August 2019. Link: https://pdg.lbl.gov/2020/listings/rpp2020-list-neutrino-prop.pdf. 3.1.3

[49] E. Elfgren and S. Fredriksson. Mass limits for heavy neutrinos. Astronomy and Astrophysics, 479(2):347-353, December 2007. Link: https://www.aanda.org/articles/aa/pdf/2008/08/aa889807.pdf. 3.1 .3

[50] Mark P. Hertzberg. Structure Formation in the Very Early Universe. Physics Magazine, 13(26), February 2020. Link: https://physics.aps.org/articles/v13/16. 3.3.3

[51] N. G. Busca, T. Delubac, J. Rich, S. Bailey, A. Font-Ribera, D. Kirkby, J.-M. Le Goff, M. M. Pieri, A. Slosar, E. Aubourg, et al. Baryon acoustic oscillations in the Lya forest of BOSS quasars. Astronomy and Astrophysics, 552(A96), April 2013. Links: https://www.aanda.org/2013-highlights/914-baryon-acoustic-oscillations-in-thelyman-alpha-forest-of-boss-quasars-busca-et-al and https://arxiv.org/abs/1211.2616. 3.3.3 
[52] S. Perlmutter, G. Aldering, G. Goldhaber, R. A. Knop, P. Nugent, P. G. Castro, S. Deustua, S. Fabbro, A. Goobar, Groom, et al. Measurements of $\Omega$ and $\Lambda$ from 42 high-redshift supernovae $\Omega$. Astrophysical Journal, 517(2):565-586, June 1999 . Link: https://iopscience.iop.org/article/10.1086/307221/meta. 3.3.3

[53] Adam G. Riess, Alexei V. Filippenko, Peter Challis, Alejandro Clocchiatti, Alan Diercks, Peter M. Garnavich, Ron L. Gilliland, Craig J. Hogan, Saurabh Jha, Robert P. Kirshner, et al. Observational Evidence from Supernovae for an Accelerating Universe and a Cosmological Constant. Astronomical Journal, 116(3):1009-1038, September 1998. Link: https://iopscience.iop.org/article/10.1086/300499/meta. 3.3.3

[54] Adam G. Riess, Louis-Gregory Strolger, John Tonry, Stefano Casertano, Henry C. Ferguson, Bahram Mobasher, Peter Challis, Alexei V. Filippenko, Saurabh Jha, Weidong Li, et al. Type Ia Supernova Discoveries at $\mathrm{z}>1$ from the Hubble Space Telescope: Evidence for Past Deceleration and Constraints on Dark Energy Evolution. Astrophysical Journal, 607(2):665-687, June 2004. Link: http://iopscience.iop.org/0004-637X/607/2/665. 3.3.3

[55] Natalie Wolchover. New Wrinkle Added to Cosmology's Hubble Crisis. Quanta Magazine, February 2020. Link: https://www.quantamagazine.org/new-wrinkle-added-to-cosmologys-hubble-crisis$20200226 / .3 .3 .3$

[56] Wendy L. Freedman, Barry F. Madore, Taylor Hoyt, In Sung Jang, Rachael Beaton, Myung Gyoon Lee, Andrew Monson, Jill Neeley, and Jeffrey Rich. Calibration of the Tip of the Red Giant Branch (TRGB). Astrophysical Journal, 891(1):57, March 2020. Link: https://iopscience.iop.org/article/10.3847/1538-4357/ab7339. 3.3.3

[57] Vivian Poulin, Tristan L. Smith, Tanvi Karwal, and Marc Kamionkowski. Early Dark Energy can Resolve the Hubble Tension. Physical Review Letters, 122(22):221301, June 2019. Link: https://link.aps.org/doi/10.1103/PhysRevLett.122.221301. 3.3.3

[58] Charlie Wood. A New Cosmic Tension: The Universe Might Be Too Thin. Quanta Magazine, September 2020. Link: https://www.quantamagazine.org/a-new-cosmic-tension-the-universe-mightbe-too-thin-20200908/. 3.4

[59] Khaled Said, Matthew Colless, Christina Magoulas, John R. Lucey, and Michael J. Hudson. Joint analysis of 6dFGS and SDSS peculiar velocities for the growth rate of cosmic structure and tests of gravity. Monthly Notices of The Royal Astronomical Society, 497(1):1275-1293, July 2020. Link: https://academic.oup.com/mnras/articleabstract $/ 497 / 1 / 1275 / 5870121$ ? redirectedFrom=fulltext. 3.4

[60] Supranta S. Boruah, Michael J. Hudson, and Guilhem Lavaux. Cosmic flows in the nearby Universe: new peculiar velocities from SNe and cosmological constraints. Monthly Notices of The Royal Astronomical Society, August 2020. Link: https://academic.oup.com/mnras/advance-articleabstract/doi/10.1093/mnras/staa2485/5894929? redirectedFrom=fulltext. 3.4

[61] Whitney Clavin. Rotating Galaxies Galore. April $2020 . \quad$ Link: https://www.caltech.edu/about/news/rotating-galaxies-galore. 3.7

[62] O. LeFevre, M. Bethermin, A. Faisst, P. Capak, P. Cassata, J. D. Silverman, D. Schaerer, and L. Yan. The ALPINE-ALMA [CII] survey: Survey strategy, observations and sample properties of 118 starforming galaxies at $4<\mathrm{z}<6$. October 2019. Link: https://doi.org/10.1051/0004-6361/201936965. 3.7

[63] Joshua D. Simon and Marla Geha. The Kinematics of the Ultra-faint Milky Way Satellites: Solving the Missing Satellite Problem. Astrophys. J., 670(1):313-331, November 2007. Link: https://iopscience.iop.org/article/10.1086/521816. 3.7

[64] Ana Bonaca, David W. Hogg, Adrian M. Price-Whelan, and Charlie Conroy. The Spur and the Gap in GD-1: Dynamical Evidence for a Dark Substructure in the Milky Way Halo. Astrophysical Journal, 880(1):38, July 2019. Link: https://iopscience.iop.org/article/10.3847/1538-4357/ab2873. 3.7

[65] David Ehrenstein. Mapping Dark Matter in the Milky Way. Physics Magazine, 12(51), May 2019. Link: https://physics.aps.org/articles/v12/51. 3.7 
[66] Ben Forrest, Marianna Annunziatella, Gillian Wilson, Danilo Marchesini, Adam Muzzin, M. C. Cooper, Z. Cemile Marsan, Ian McConachie, Jeffrey C. C. Chan, Percy Gomez, et al. An Extremely Massive Quiescent Galaxy at $\mathrm{z}=3.493$ : Evidence of Insufficiently Rapid Quenching Mechanisms in Theoretical Models. Astrophysical Journal, 890(1):L1, February 2020. Link: https://iopscience.iop.org/article/10.3847/2041-8213/ab5b9f. 3.8

[67] Katherine E. Whitaker, Christina C. Williams, Lamiya Mowla, Justin S. Spilker, Sune Toft, Desika Narayanan, Alexandra Pope, Georgios E. Magdis, Pieter G. van Dokkum, Mohammad Akhshik, et al. Quenching of star formation from a lack of inflowing gas to galaxies. Nature, 597(7877):485-488, September 2021. Link: https://doi.org/10.1038/s41586-021-03806-7. 3.8

[68] David A. Buote and Aaron J. Barth. The Extremely High Dark Matter Halo Concentration of the Relic Compact Elliptical Galaxy Mrk 1216. Astrophysical Journal, 877(2):91, May 2019. Link: https://iopscience.iop.org/article/10.3847/1538-4357/ab1008. 3.8

[69] M. Markevitch, A. H. Gonzalez, D. Clowe, A. Vikhlinin, W. Forman, C. Jones, S. Murray, and W. Tucker. Direct Constraints on the Dark Matter Self-Interaction Cross Section from the Merging Galaxy Cluster 1E 0657-56. Astrophysical Journal, 606(2):819-824, May 2004. Link: https://iopscience.iop.org/article/10.1086/383178. 4.2

[70] Marvin Holten, Luca Bayha, Keerthan Subramanian, Carl Heintze, Philipp M. Preiss, and Selim Jochim. Observation of Pauli Crystals. Physical Review Letters, 126:020401, January 2021. Link: https://link.aps.org/doi/10.1103/PhysRevLett.126.020401. 4.3.2

[71] Christie Chiu. Revealing a Pauli Crystal. Physics, 15(5), January 2021. Link: https://physics.aps.org/articles/v14/5. 4.3.2

[72] L. Gurung, T. J. Babij, S. D. Hogan, and D. B. Cassidy. Precision Microwave Spectroscopy of the Positronium $n=2$ Fine Structure. Physical Review Letters, 125:073002, August 2020. Link: https://link.aps.org/doi/10.1103/PhysRevLett.125.073002. 4.3.4

[73] Matteo Rini. A Fine Positronium Puzzle. Physics, 13, August 2020. Link: https://physics.aps.org/articles/v13/s99. 4.3.4

[74] V. M. Abazov, B. Abbott, M. Abolins, B. S. Acharya, M. Adams, T. Adams, M. Agelou, J.-L. Agram, S. H. Ahn, M. Ahsan, et al. Search for right-handed $W$ bosons in top quark decay. Physical Review D, 72:011104, July 2005. Link: https://link.aps.org/doi/10.1103/PhysRevD.72.011104. 4.4.2

[75] Paul Langacker and S. Uma Sankar. Bounds on the mass of W sub R and the W sub L - W sub R mixing angle. zeta. in general $\mathrm{SU}(2)$ sub L times $\mathrm{SU}(2)$ sub $\mathrm{R}$ times $\mathrm{U}(1)$ models. Physical Review $D, 40(5): 1569-1585$, September 1989. Link: https://inspirehep.net/literature/277249. 4.4.2

[76] G. A. Gonzalez-Sprinberg and J. Vidal. Tau magnetic moment. Proceedings of The International Conference On Nanoscience and Technology, 912(1):012001, 2017. Link: http://stacks.iop.org/1742$6596 / 912 / \mathrm{i}=1 / \mathrm{a}=012001.4 .5 .3$

[77] Thomas J. Buckholtz. Models for Physics of the Very Small and Very Large, volume 14 of Atlantis Studies in Mathematics for Engineering and Science. Springer, 2016. Series editor: Charles K. Chui. Link: https://link.springer.com/book/10.2991/978-94-6239-166-6. 5

[78] Thomas J. Buckholtz. Predict particles beyond the standard model; then, narrow gaps between physics theory and data. In Proceedings of the 9th Conference on Nuclear and Particle Physics (19-23 Oct. 2015 Luxor-Aswan, Egypt), May 2016. Link: http://www.afaqscientific.com/nuppac15/npc1509.pdf. 5

Copyright (c) 2021 Thomas J. Buckholtz

Manuscript date: December 26, 2021 